\title{
Taxonomical revision of the Longitarsus capensis species-group: An example of Mediterranean-southern African disjunct distributions (Coleoptera: Chrysomelidae)
}

\author{
Maurizio BIONDI and PaOla D’ALESSANDRO \\ Dipartimento di Scienze Ambientali, University of L’Aquila, 67010 Coppito - L'Aquila, Italy, e-mails: biondi@univaq.it, \\ paola.dalessandro@univaq.it
}

Key words. Chrysomelidae, Alticinae, Boraginaceae, Afrotropical region, Mediterranean area, new species, disjunct distribution

\begin{abstract}
Southern-African Longitarsus capensis species-group, which is closely related to the Mediterranean anchusae speciesgroup is revised. L. capensis species-group includes 15 species, 8 of which are new to science: Longitarsus capensis Baly, $1877, L$. cedarbergensis Biondi, 1999, L. luctuosus Biondi, 1999, L. lugubris Biondi, 1999, L. melanicus Biondi, 1999, L. neseri Biondi, 1999, L. transvaalensis Biondi, 1999, L. afromeridionalis sp.n., L. debiasei sp.n., L. grobbelaariae sp.n., L. hexrivierbergensis sp.n., L. malherbei sp.n., L. piketbergensis sp.n., L. rouxi sp.n., and L. sudafricanus sp.n. A key to all the species is presented as well as line drawings of male and female genitalia, scanning electron micrographs of some diagnostic morphological characters, and autoecological and zoogeographical data. Relationships within the capensis-group, and between the anchusae and capensis-group are better defined and hypotheses explaining the separate distributions of Mediterranean and south African anchusae and capensis species-groups proposed. Finally, the host-plant shift from native Lobostemon spp. to the introduced plant Echium plantagineum L. shown by some species of the capensis group is also discussed.
\end{abstract}

\section{INTRODUCTION}

Longitarsus Latreille, as described in Berthold (1827), is a very large flea beetle genus (Chrysomelidae, Alticinae), which occurs throughout all zoogeographical regions, and includes about 600 known species.

In the Mediterranean area, where Longitarsus is better known from an autecological standpoint, some speciesgroups, such as aeneus, anchusae, exsoletus and echii, are closely associated with Boraginaceae and generally show trophic specialization (Jolivet \& Hawkeswood, 1995; Biondi, 1996): $34 \%$ of Longitarsus species are monophagous (feed on one or two closely related botanical genera), 56\% oligophagous (feed on more botanical genera of one or two closely related families) and only $10 \%$ polyphagous (feed on many botanical species not closely related). Therefore, at least in the Mediterranean area, $90 \%$ of the Longitarsus species associated with Boraginaceae (34\% monophagous and 56\% oligophagous) feed exclusively on them (Biondi, 1996). The trophic categories are those of Biondi (1996) and Fernandez \& Hilker (2007).

In the Afrotropical Region, the genus Longitarsus presently includes about 120 described species, but many other yet undescribed taxa exist. Autecological data for Afrotropical species are extremely scarce or completely lacking compared to the Mediterranean area. In the literature there are only a few reports of Longitarsus species associated with Boraginaceae, namely Longitarsus punctifrons Weise, 1895 (= gossypii Bryant, 1941) collected on Heliotropium sp. (Furth, 1985), and species of the capensis group collected on Lobostemon spp., Anchusa capensis Thumb. and Echium plantagineum L. (Biondi, 1999).

This paper includes the 15 species that are now attributed to the capensis group (Figs 1-15), including eight new species: Longitarsus capensis Baly, 1877; L. cedarbergensis Biondi, 1999; L. luctuosus Biondi, 1999; L. lugubris Biondi, 1999; L. melanicus Biondi, 1999; L. neseri Biondi, 1999; L. transvaalensis Biondi, 1999; L. afromeridionalis sp.n.; L. debiasei sp.n.; L. grobbelaariae sp.n.; L. hexrivierbergensis sp.n.; L. malherbei sp.n.; L. piketbergensis sp.n.; L. rouxi sp.n.; and L. sudafricanus sp.n.

As reported in Biondi (1999), the South African species of the capensis-group are closely related to the Western-Palaearctic, especially Mediterranean, species of the anchusae-group (sensu Biondi, 1995). Both these Longitarsus species-groups, containing exclusively species with a black integument, are strictly associated with Boraginaceae and share the following updated morphological characteristics that differentiate the anchusae and capensis groups within Longitarsus: (i) head with impunctate vertex and frons with some large punctures impressed near the frontal grooves; (ii) elytra apically subtruncate or widely and independently rounded; (iii) humeral callus always completely absent in capensisgroup, rarely developed in anchusae-group; (iv) hind wings strongly reduced (species always subapterous in capensis-group; brachypterous, sub-brachypterous or very rarely macropterous in anchusae-group); (v) large spermatheca (generally longer than $0.30 \mathrm{~mm}$ ) with ducts often widely arcuate and usually uncoiled or with one coil, very rarely with two coils; (vi) median lobe of 
aedeagus strongly sclerotized, mostly with a distinct apical median small tooth and a ventral sulcus invariably with clear impressions; (vii) impressed elytral punctation generally dense and without signs of striae even in sutural area. The latter character is very important for distinguishing the species of the anchusae and capensis groups from the black species of Longitarsus associated with Lamiaceae, common in both the Mediterranean area and southern Africa.

Further taxonomical, zoogeographical and autecological data on the species of the Longitarsus capensisgroup were collected during zoological collecting trips that were part of an Italian research project (PRIN 2004057217) aimed at interpreting the disjunct distribution of different plant and animal groups in the Mediterranean-South African regions (cf. Axerold \& Raven, 1978; Balinsky, 1962; Coleman et al., 2003; Jürgens, 1997; La Greca, 1970, 1990).

\section{MATERIAL AND METHODS}

Material consisted of preserved, dried insects courteously supplied by the institutions listed below. Specimens were examined and dissected using WILD MZ8 and MZ12.5 binocular microscopes. Photomicrographs were taken using a Leica DFC500 camera. Scanning electron micrographs were taken using a PHILIPS SEM XL30 CP. Line drawings were done using the graphic software Corel-DRAW version 12. Geographical coordinates of the localities are reported in degrees, minutes and decimal minutes (DMD-WGS84); those included in square brackets were added by the authors.

\section{Abbreviations}

Morphology: LAED - length of median lobe of aedeagus; LAN - length of antennae; LB - total length of body; LE length of elytra; LP - length of pronotum; LSP - length of spermatheca; WE - width of elytra; WP - width of pronotum.

\section{Collections and depositories}

BAQ - collection of M. Biondi, Dipartimento di Scienze Ambientali, University of L'Aquila, Italy; BMNH - The Natural History Museum, London, UK; MCZR: Civic Museum of Zoology of Rome, Italy; SANC - South African National Collection, Plant Protection Research Institute, Pretoria, Gauteng, Republic of South Africa; TMSA - Transvaal Museum, Pretoria, Gauteng, Republic of South Africa; ZMHB - Museum für Naturkunde der Humboldt-Universität, Berlin, Germany.

\section{LIST OF SPECIES}

\section{Longitarsus afromeridionalis sp.n.}

Type material. Holotype $\delta$, Republic of South Africa, Western Cape Province, Road Quoin Point-Cape Agulhas, $34^{\circ} 42.186^{\prime} \mathrm{S}, 19^{\circ} 42.667^{\prime} \mathrm{E}, 111 \mathrm{~m}, 21 . i x .2006$ (fynbos), on Lobostemon cf. lucidus, M. Biondi \& A. De Biase leg. (SANC). Paratypes: same data, $38 \hat{0}, 33 \uparrow$ (BAQ, SANC).

Diagnosis. L. afromeridionalis sp.n. is externally easily distinguishable from the other species of the capensisgroup mainly by the following morphological characters: size bigger $(\mathrm{LB}=2.28 \mathrm{~mm}$ in males and $=2.43 \mathrm{~mm}$ in females); pronotum weakly transverse (WP/LP $=1.35$ in males and $\mathrm{WP} / \mathrm{LP}=1.37$ in females), laterally very slightly rounded; antennae shorter $[\mathrm{LAN} /(\mathrm{LE}+\mathrm{LP})=0.75$ in males and $=0.72$ in females]; legs reddish, with front and middle femora not darkened. Median lobe of aedeagus (Fig. 29) closely resembles that of L. sudafricanus sp.n. (Fig. 23), while the large spermatheca (Figs $31 \mathrm{a}-\mathrm{c}$ ) with widely arcuated duct [very similar to that of L. anchusae (Biondi, 1995: Fig. 9)] places L. afromeridionalis sp.n. in a rather isolated taxonomical position among the Afrotropical species of this group.

Description. Holotype ( $\delta$ dorsal integument black, with evident metallic reflection. Body (Fig. 1) moderately elongate-oval $(\mathrm{LB}=2.30 \mathrm{~mm})$, rather convex. Maximum pronotal width in middle: $0.78 \mathrm{~mm}$; maximum elytral width in middle: $1.13 \mathrm{~mm}$.

Head with impunctate vertex and frons but with some coarse and large punctures near frontal grooves; surface wrinkled and sparsely punctulated between eyes. Frontal tubercles weakly delimited, short, oblique, with smooth surface; frontal grooves finely impressed, distally divergent from ocular margin; frontal carina wide, weakly raised; labrum sub-trapezoidal, blackened; palpi strongly darkened; eyes sub-elliptical, normal size; antennae with yellowish segments 1-4 and gradually darkened segments $5-11$; length of each segment of right antenna proportional to numerical sequence $25: 15: 12: 16: 18: 16$ : $18: 18: 18: 18: 22(1=0.032 \mathrm{~mm})$.

Pronotum scarcely transverse $(\mathrm{LP}=0.60 \mathrm{~mm}$; WP/LP $=$ 1.29 ), laterally very weakly rounded, basally slightly narrower; lateral and basal margin very finely bordered; punctation evenly distributed with rather little and densely impressed punctures on a very finely reticulate surface. Scutellum wide, semi-circular, with smooth surface.

Elytra elongate $(\mathrm{LE}=1.53 \mathrm{~mm}$; LE/LP $=2.54)$, laterally rounded, apically slightly obliquely sub-truncate; pygidium partially uncovered; punctation similar to that on pronotum but slightly sparser, very homogenous, with small punctures impressed on a smooth surface; humeral calli absent; subapterous wings.

Legs with reddish tibiae, tarsi, and anterior and middle femora; hind femora dorsally dark in colour; ungual segments distinctly darkened; distal two thirds of hind tibiae dentate on external edge; apical spur of hind tibiae long about $1 / 2$ of tibial apex width, reddish; first anterior and middle tarsomeres slightly dilated.

Ventral part entirely black; last sternite without dimples or special preapical impressions.

Median lobe of aedeagus (Fig. 29) weakly elongate $(\mathrm{LAED}=0.89 \mathrm{~mm}$; LE/LAED $=1.72)$, rather thickset, in ventral view medially slightly narrower; apical part sublanceolate, with an apically sub-rounded median small tooth; ventral sulcus elongate, gradually narrowing from apex towards basal part, basally not interrupted; dorsal sulcus V-shaped, apical half visible; dorsal ligula very narrow; in lateral view basal $2 / 3$ s of median lobe curved, then straight and apically clearly sinuous.

Variation. of $(\mathrm{n}=10$; mean and standard deviation): $\mathrm{LE}=1.54 \pm 0.10 \mathrm{~mm} ; \mathrm{WE}=1.15 \pm 0.07 \mathrm{~mm} ; \mathrm{LP}=0.57$ $\pm 0.03 \mathrm{~mm} ; \mathrm{WP}=0.74 \pm 0.04 \mathrm{~mm} ; \mathrm{LAN}=1.56 \pm 0.09$ $\mathrm{mm} ; \mathrm{LAED}=0.87 \pm 0.05 \mathrm{~mm} ; \mathrm{LB}=2.54 \pm 0.25 \mathrm{~mm}$; $\mathrm{LE} / \mathrm{LP}=2.69 \pm 0.09 ; \mathrm{WE} / \mathrm{WP}=1.55 \pm 0.06 ; \mathrm{WP} / \mathrm{LP}=$ 


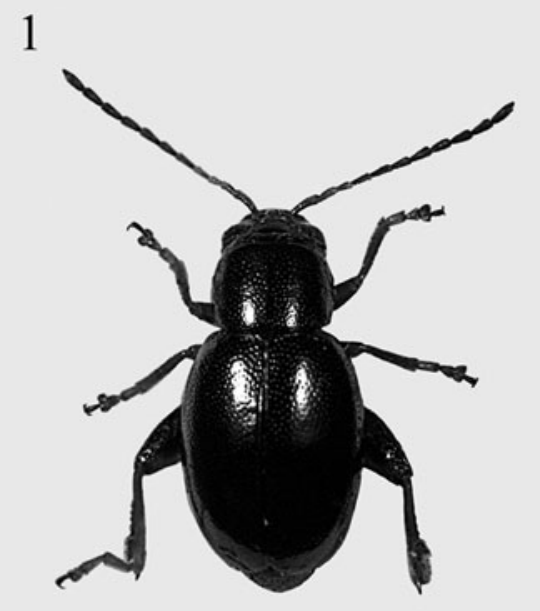

$\mathrm{LE}=1.65 \mathrm{~mm}$

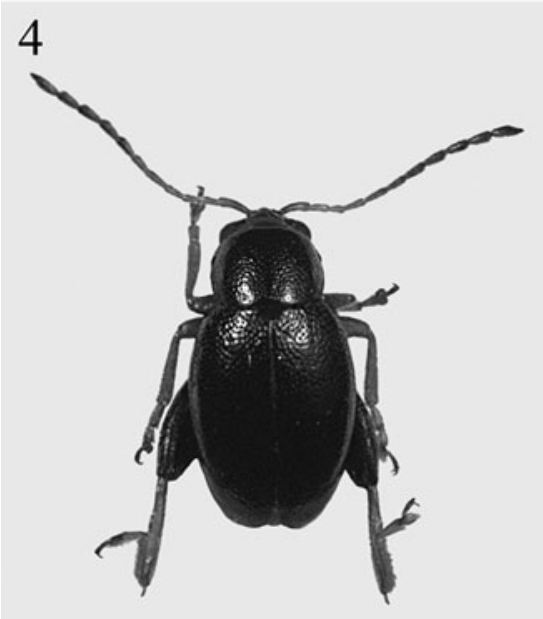

$\mathrm{LE}=1.39 \mathrm{~mm}$

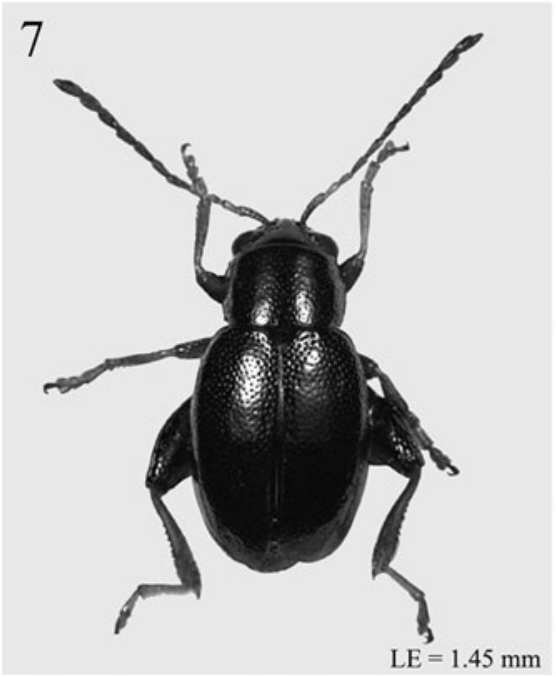

2

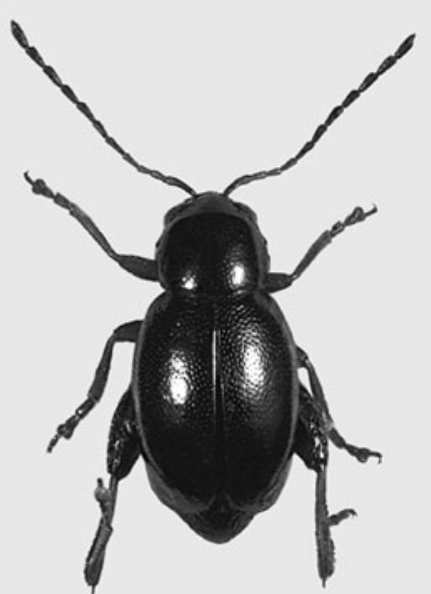

$\mathrm{LE}=1.50 \mathrm{~mm}$

5

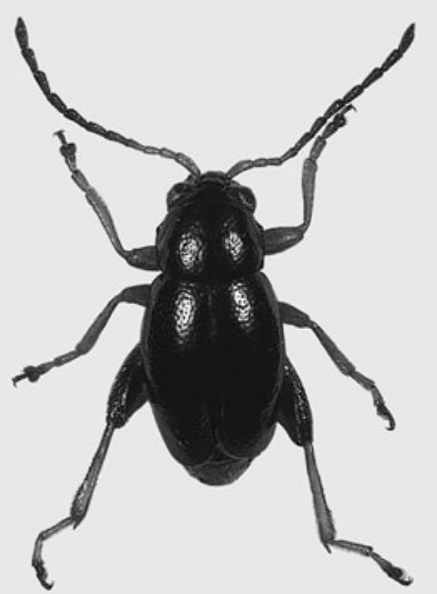

$\mathrm{LE}=1.20 \mathrm{~mm}$

\section{8}

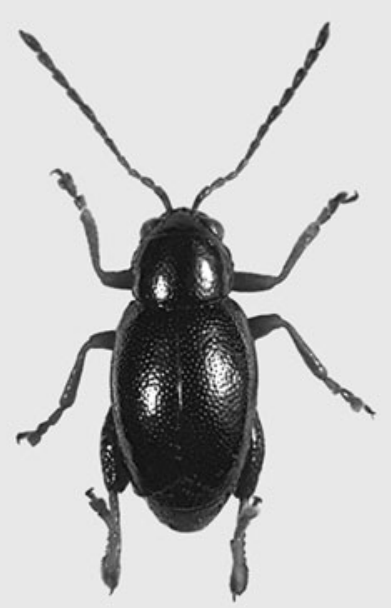

3

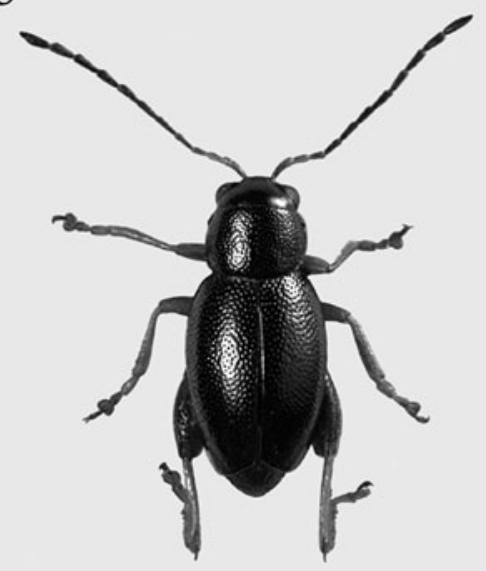

$\mathrm{LE}=1.28 \mathrm{~mm}$

6

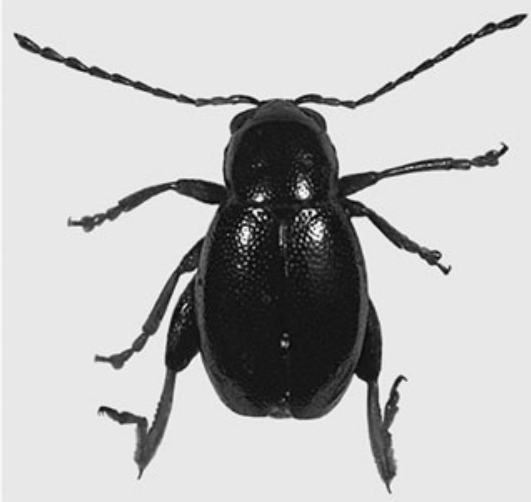

$\mathrm{LE}=1.38 \mathrm{~mm}$

9

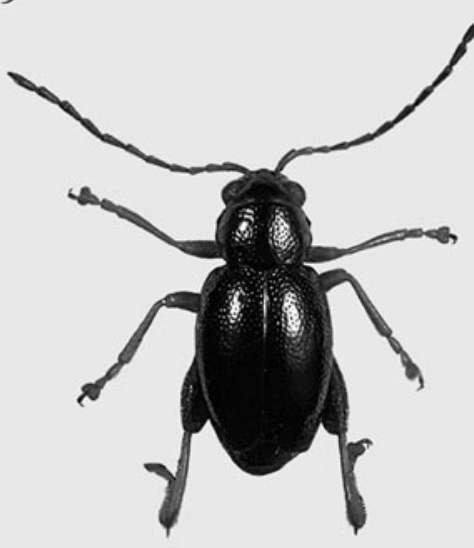

$\mathrm{LE}=1.15 \mathrm{~mm}$

$\mathrm{LE}=1.10 \mathrm{~mm}$

Figs 1-9. Habitus of Longitarsus capensis species-group: L. afromeridionalis sp.n. (1); L. capensis Baly (2); L. cedarbergensis Biondi (3); L. debiasei sp.n. (4); L. grobbelaariae sp.n. (5); L. hexrivierbergensis sp.n. (6); L. luctuosus Biondi (7); L. lugubris Biondi (8); L. malherbei sp.n. (9).

$1.30 \pm 0.03 ; \mathrm{LE} / \mathrm{LAED}=1.77 \pm 0.06 ; \mathrm{LAN} /(\mathrm{LE}+\mathrm{LP})=$ $0.74 \pm 0.01$. ㅇ $(\mathrm{n}=10$; mean and standard deviation $)$ : $\mathrm{LE}$ $=1.72 \pm 0.08 \mathrm{~mm} ; \mathrm{WE}=1.28 \pm 0.05 \mathrm{~mm} ; \mathrm{LP}=0.61 \pm$ $0.03 \mathrm{~mm} ; \mathrm{WP}=0.80 \pm 0.03 \mathrm{~mm} ; \mathrm{LAN}=1.60 \pm 0.12 \mathrm{~mm}$;
$\mathrm{LSP}=0.43 \pm 0.03 \mathrm{~mm} ; \mathrm{LB}=2.81 \pm 0.20 \mathrm{~mm} ; \mathrm{LE} / \mathrm{LP}=$ $2.83 \pm 0.06 ; \mathrm{WE} / \mathrm{WP}=1.59 \pm 0.03 ; \mathrm{WP} / \mathrm{LP}=1.32 \pm$ $0.03 ; \mathrm{LE} / \mathrm{LSP}=4.03 \pm 0.26 ; \mathrm{LAN} /(\mathrm{LE}+\mathrm{LP})=0.68 \pm$ 0.03 . 
Paratypes very similar in shape, sculpture and colour to the holotype, but sometimes with more or less darkened hind femora and distal tarsomeres. Females generally bigger than males, with slightly shorter antennae and not enlarged first anterior and middle tarsomeres. Spermatheca (Figs 31a-c) larger than in the other species of the capensis-group, with sub-reniform, sometimes subcylindrical, basal part; distal part well developed, moderately distinct from basal part; duct clearly elongate, basally or sub-basally inserted, uncoiled, generally widely arcuate.

Etymology. The name of this new species refers to the geographic region where it lives and means "from southern Africa".

Distribution. Southern-western coastal area of the Western Cape Province (Republic of South Africa) (Fig. 56). SouthernWestern Afrotropical chorotype (cf. Biondi \& D'Alessandro, 2006)

Host plants. Lobostemon cf. lucidus (Lehm.) H. Bueck (Boraginaceae).

\section{Longitarsus capensis Baly}

Longitarsus capensis Baly, 1877: 292; Biondi, 1999: 517.

Material examined. Republic of South Africa: "Cape of

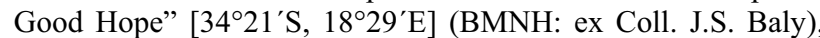
1 to (lectotype des. M. Biondi, 1999); Western Cape Province: Cape Town, Table Mountain, 150-500 m, 13 0 , 23 (BAQ; MCZR); $12 \mathrm{~km}$ ESE of Langabaan (R27), $30 \mathrm{~m}, 7 \hat{0}, 1$ ㅇ (BAQ); Cape Peninsula, 0-150 m, 5ठ, 10 으 (BAQ, ZMHB); 2 $\mathrm{km}$ N Stellenbosch, $200 \mathrm{~m}, 30$, 4 ㅇ (BAQ); Lion's Head, 150-550 m, 7ठิ, 9우 (BAQ); Malmesbury, $93 \mathrm{~m}, 5 \hat{0}, 6$ (BAQ); Du Toitskloof Pass, SW slope, $286 \mathrm{~m}, 10 \AA, 16 ㅇ$ (BAQ). Phenology recorded: July-October.

Distribution. Western part of the Western Cape Province (Republic of South Africa) (Fig. 56). This distribution can be attributed to the Southern-Western Afrotropical chorotype, as proposed by Biondi \& D'Alessandro (2006).

Host plants. Lobostemon fruticosus (L.) H. Buek, L. cf. lucidus, Anchusa capensis Thumb. and Echium plantagineum L. (Boraginaceae).

\section{Longitarsus cedarbergensis Biondi}

Longitarsus cedarbergensis Biondi, 1999: 519.

Material examined. Republic of South Africa: Western Cape Province, road Clanwilliam-Pakhuis Pass $\left[32^{\circ} 08^{\prime} \mathrm{S}, 18^{\circ} 56^{\prime} \mathrm{E}\right]$, 200-900 m, 11.ix.1994 (fynbos and xeric grassland), P. Audisio, M. Biondi \& M. Bologna leg., 10 (holotype) (BAQ); ditto, 2 우 (with eggs) (paratypes) (BAQ); Western Cape Province, Pakhuis Pass, $32^{\circ} 08.898^{\prime} \mathrm{S}, 1^{\circ} 01.993^{\prime} \mathrm{E}, 883 \mathrm{~m}, 14.1 x .2006$ (fynbos), on Lobostemon cf. dorotheae, M. Biondi \& A. De Biase leg., 10, 2 ㅇ (BAQ).

Distribution. Cedarberg area (Republic of South Africa: Western Cape Province) (Fig. 56). Southern-Western Afrotropical chorotype (cf. Biondi \& D'Alessandro, 2006).

Host plants. Lobostemon cf. dorotheae M.H. Buys (Boraginaceae).

\section{Longitarsus debiasei sp.n.}

Type material. Holotype $\hat{\delta}$, Republic of South Africa, Western Cape Province, road Ashton-Montagu, Kogmanskloof, $33^{\circ} 49.436^{\prime} \mathrm{S}, 20^{\circ} 05.417^{\prime} \mathrm{E}, 180 \mathrm{~m}, 15 . x .2005$, on Echium plantagineum, P. Audisio, M. Biondi \& A. De Biase leg. (SANC). Paratypes: same locality, date, host plant and collectors as the holotype, 10ิ, 1 ( (BAQ, SANC); Republic of South Africa, ditto, $33^{\circ} 50.668^{\prime} \mathrm{S}, 20^{\circ} 05.378^{\prime} \mathrm{E}, 195$ m, 9.x.2005, on Echium plantagineum, P. Audisio, M. Biondi \& A. De Biase leg., 40, 2 우 (BAQ).

Diagnosis. This new species is externally very similar to L. hexrivierbergensis sp.n. from which it differs in the colour of its legs and antennae, slightly paler in L. debiasei sp.n., and its elytral apex, which is not obliquely subtruncate. These two new species are easily distinguishable by the shapes of the median lobe of the aedeagus and the spermatheca (Figs 17, 21, 35a-b, 36a-b). On the basis of the median lobe of the aedeagus, L. debiasei sp.n. shows affinities with L. sudafricanus sp.n. (Fig. 23), while the spermateca, with uncoiled duct, is very similar that of $L$. cedarbergensis and L. transvaalensis (Figs 40a-b, 41).

Description. Holotype ( $\hat{\sigma})$ dorsal integument black, with evident greenish metallic reflection. Body (Fig. 4) elongate-oval $(\mathrm{LB}=1.86 \mathrm{~mm})$, moderately convex. Maximum pronotal width in middle: $0.65 \mathrm{~mm}$; maximum elytral width in middle: $0.98 \mathrm{~mm}$.

Head with impunctate vertex; frons with fine punctulation impressed on a clearly wrinkled surface. Frontal tubercles weakly delimited, elongate, oblique, with smooth surface; frontal grooves finely impressed, distally divergent from ocular margin; frontal carina moderately wide, weakly raised; labrum sub-trapezoidal, clearly blackened; palpi strongly darkened; eyes sub-elliptical, normal size; antennae with yellowish segments 1-5 and gradually darkened segments 6-11; length of each segment of right antenna proportional to numerical sequence $24: 14: 12: 15: 19: 16: 19: 18: 19: 19: 25(1=0.032$ $\mathrm{mm})$.

Pronotum weakly transverse $(\mathrm{LP}=0,50 \mathrm{~mm}$; WP/LP $=$ 1.30) laterally sub-parallel, basally a little narrower; lateral and basal margin finely bordered; punctation uniformly distributed with densely and strongly impressed small punctures on a microreticulate surface. Scutellum wide, semi-circular, with weakly reticulate surface.

Elytra elongate $(\mathrm{LE}=1.39 \mathrm{~mm} ; \mathrm{LE} / \mathrm{LP}=2.78)$, laterally rounded, apically sub-truncate; pygidium partially uncovered; punctation similar to that on pronotum one but with punctures slightly larger, impressed on a weakly wrinkled surface; humeral calli absent; subapterous wings.

Legs with yellowish tibiae and tarsi; anterior and middle femora basally partially and very weakly darkened; hind femora dark; distal three quarters of hind tibiae dentate on external edge; apical spur of hind tibiae long about $3 / 5$ of tibial apex width, reddish; first anterior and middle tarsomeres slightly dilated.

Ventral part entirely black; last sternite without dimples or special preapical impressions.

Median lobe of aedeagus (Fig. 17) moderately elongate $(\mathrm{LAED}=0.80 \mathrm{~mm}$; LE/LAED = 1.73), rather slender, in ventral view middle slightly narrower; apical part laterally sub-rounded, distally with an apically rounded small median tooth; ventral sulcus elongate, basal third clearly narrower and basally not interrupted; dorsal sulcus $\mathrm{V}$-shaped, apical third well impressed; dorsal ligula very narrow; in lateral view basal $3 / 4$ s of median lobe strongly curved and apex slightly bent in ventral direction. 

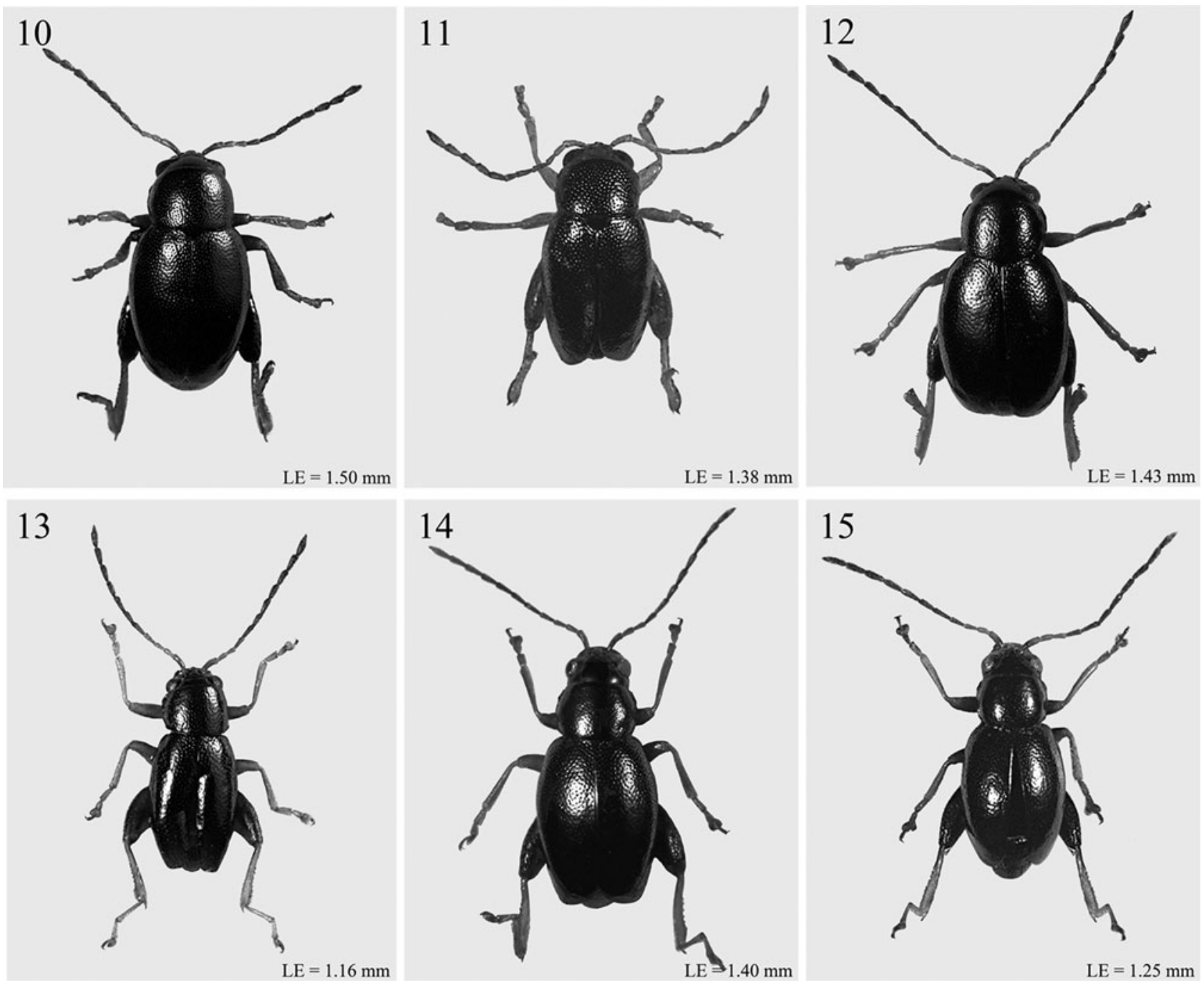

Figs 10-15. Habitus of Longitarsus capensis species-group: L. melanicus Biondi (10); L. neseri Biondi (11); L. piketbergensis sp.n. (12); L. rouxi sp.n. (13); L. sudafricanus sp.n. (14); L. transvaalensis Biondi (15).

Variation. ơ $(\mathrm{n}=6$; mean and standard deviation): LE $=1.44 \pm 0.04 \mathrm{~mm} ; \mathrm{WE}=1.03 \pm 0.04 \mathrm{~mm} ; \mathrm{LP}=0.54 \pm$ $0.03 \mathrm{~mm} ; \mathrm{WP}=0.70 \pm 0.03 \mathrm{~mm} ; \mathrm{LAN}=1.60 \pm 0.05 \mathrm{~mm}$ $\mathrm{LAED}=0.81 \pm 0.01 \mathrm{~mm} ; \mathrm{LB}=2.05 \pm 0.14 \mathrm{~mm} ; \mathrm{LE} / \mathrm{LP}=$ $2.68 \pm 0.12 ; \mathrm{WE} / \mathrm{WP}=1.47 \pm 0.03 ; \mathrm{WP} / \mathrm{LP}=1.30 \pm$ $0.05 ; \mathrm{LE} / \mathrm{LAED}=1.79 \pm 0.07 ; \mathrm{LAN} /(\mathrm{LE}+\mathrm{LP})=0.81 \pm$ 0.02 . $+(\mathrm{n}=3$; mean and standard deviation $): \mathrm{LE}=1.60$ $\pm 0.07 \mathrm{~mm} ; \mathrm{WE}=1.12 \pm 0.04 \mathrm{~mm} ; \mathrm{LP}=0.53 \pm 0.01 \mathrm{~mm}$; $\mathrm{WP}=0.73 \pm 0.02 \mathrm{~mm} ; \mathrm{LAN}=1.61 \pm 0.01 \mathrm{~mm} ; \mathrm{LSP}=$ $0.31 \pm 0.01 \mathrm{~mm} ; \mathrm{LB}=2.42 \pm 0.07 \mathrm{~mm} ; \mathrm{LE} / \mathrm{LP}=3.00 \pm$ $0.08 ; \mathrm{WE} / \mathrm{WP}=1.52 \pm 0.08 ; \mathrm{WP} / \mathrm{LP}=1.38 \pm 0.03$; $\mathrm{LE} / \mathrm{LSP}=5.12 \pm 0.23 ; \mathrm{LAN} /(\mathrm{LE}+\mathrm{LP})=0.76 \pm 0.03$.

Paratypes similar in shape, sculpture and colour to the holotype, but sometimes with weakly darkened ungual segment. Females generally a little bigger than males, with slightly shorter antennae, flatter frontal carina and first anterior and middle tarsomeres not enlarged. Spermatheca (Figs 36a-b) with sub-reniform basal part; distal part moderately developed, generally distinct from basal part; duct moderately elongate, sub-basally inserted, rather robust, uncoiled, sub-arcuate.
Etymology. The new species is named after A. De Biase (Italy, Rome), my friend and fellow traveller on many collecting trips in South Africa.

Distribution. Langeberg area (Republic of South Africa: Western Cape Province) (Fig. 57). Southern-Western Afrotropical chorotype (cf. Biondi \& D'Alessandro, 2006).

Host plants. Echium plantagineum (Boraginaceae).

\section{Longitarsus grobbelaariae sp.n.}

Type material. Holotype $\delta$, Republic of South Africa, Kwazulu-Natal, Vryheid Hill Nature Reserve, Ntinginono Eco Centre, $27^{\circ} 45.14^{\prime} \mathrm{S}, 30^{\circ} 47.11^{\prime} \mathrm{E}, 1259 \mathrm{~m}, 30$. i. $\left.-2 . i i .2007\right)$, adults beaten off flowering Heliotropium sp., E. Grobbelaar leg. (SANC). Paratypes: same locality, date, collectors and host plant as the holotype, 11 ( $\mathrm{BAQ}, \mathrm{SANC}$ ).

Diagnosis. Because of the peculiar shape of the median lobe of its aedeagus (Fig. 30), this new species occupies a rather isolated taxonomical position within the capensis species-group.

Description. Holotype ( $\widehat{c}$ ) dorsal integument black, with evident metallic reflection. Body (Fig. 5) elongateoval $(\mathrm{LB}=1.88 \mathrm{~mm})$, moderately convex. Maximum pro- 

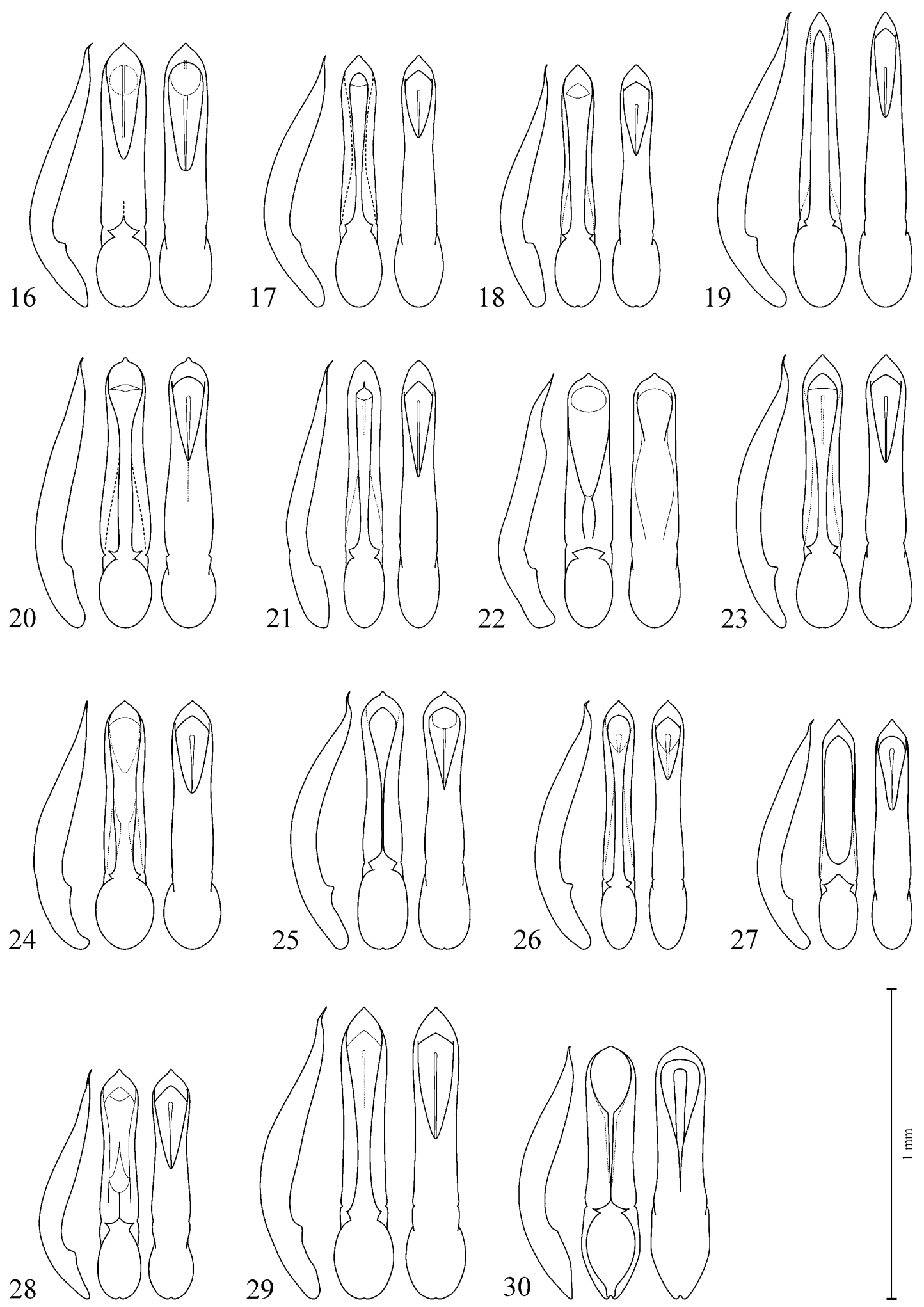

Figs 16-30. Median lobe of aedeagus in Longitarsus capensis species-group; lateral, ventral and dorsal view: L. capensis Baly, Western Cape Province, Cape Town, Lion's Head (16); L. debiasei sp.n., Western Cape Province, Kogmanskloof (17); L. transvaalensis Biondi, Eastern Transvaal, Nelshoogte Pass (18); L. neseri Biondi, Western Cape Province, Piketberg (19); L. melanicus Biondi, Western Cape Province, Danabaai (20); L. hexrivierbergensis sp.n., Western Cape Province, 35 km NE Ceres (21); L. rouxi sp.n., Western Cape Province, Cedarberg (22); L. sudafricanus sp.n., Western Cape Province, road Citrusdal-Middelberg Pass (23); L. cedarbergensis Biondi, Western Cape Province, Pakhuis Pass (24); L. luctuosus Biondi, Western Cape Province, Akkedisbergpas, neigh. Stanford (25); L. piketbergensis sp.n., Western Cape Province, Piketberg (26); L. malherbei sp.n., Western Cape Province, Algeria, Cederberg Nature Reserve (27); L. lugubris Biondi, Western Cape Province, Pakhuis Pass (28); L. afromeridionalis sp.n., Western Cape Province, Road Quoin Point-Cape Agulhas (29); L. grobbelaariae sp.n., Kwazulu-Natal, Vryheid Hill Nature Reserve (30). 
notal width in anterior third: $0.66 \mathrm{~mm}$; maximum elytral width in middle: $0.88 \mathrm{~mm}$.

Head with almost impunctate vertex and frons, but with some coarse and large punctures near frontal grooves; surface wrinkled and sparsely punctulated between eyes. Frontal tubercles finely delimited, elongate, subhorizontal, with smooth surface; frontal grooves finely impressed, distally divergent from ocular margin; frontal carina wide, apically widely rounded, weakly raised; labrum sub-trapezoidal, clearly blackened; palpi strongly darkened; eyes sub-elliptical, normal size; antennae with yellowish segments 1-3 and gradually darkened segments 4-11; length of each segment of right antenna proportional to numerical sequence $24: 16: 14: 16: 18: 16$ : $20: 19: 20: 20: 26(1=0.032 \mathrm{~mm})$.

Pronotum scarcely transverse $(\mathrm{LP}=0.51 \mathrm{~mm} ; \mathrm{WP} / \mathrm{LP}=$ 1.29), sub-trapezoidal, laterally slightly but clearly convergent from anterior angles to base; lateral and basal margin finely bordered; punctation uniformly distributed with moderately dense and weakly impressed little punctures on clearly microreticulate surface. Scutellum wide, semi-circular, with weakly reticulate surface.

Elytra elongate $(\mathrm{LE}=1.20 \mathrm{~mm}$; LE/LP $=2.34)$, laterally rounded, apically sub-truncate; pygidium partially uncovered; punctation similar to that on pronotum but with punctures slightly larger and deeper impressed on a rather smooth surface; humeral calli absent; subapterous wings.

Legs entirely yellowish but with distally browned tarsi and hind femora; distal two thirds of hind tibiae weakly dentate on external edge; apical spur of hind tibiae long about $4 / 5$ of tibial apex width, reddish; first anterior and middle tarsomeres slightly dilated.

Ventral part entirely black; last sternite without dimples or special preapical impressions.

Median lobe of aedeagus (Fig. 30) a little elongated $(\mathrm{LAED}=0.73 \mathrm{~mm}$; LE/LAED = 1.66), rather thickset, in ventral view weakly narrower in middle; apical part almost regularly rounded, distally with a very weakly prominent small median tooth; ventral sulcus elongate, strongly impressed, clearly narrower in apical third and basally interrupted; dorsal sulcus V-shaped, well impressed distally; dorsal ligula wide, apically subrounded; in lateral view, median lobe clearly and regularly curved, with apex slightly bent in ventral direction.

Variation. o $(\mathrm{n}=1)$ : $\mathrm{LE}=1.20 \mathrm{~mm} ; \mathrm{WE}=0.88 \mathrm{~mm}$; $\mathrm{LP}=0.51 \mathrm{~mm} ; \mathrm{WP}=0.66 \mathrm{~mm} ; \mathrm{LAN}=1.45 \mathrm{~mm} ; \mathrm{LAED}$ $=0.73 \mathrm{~mm} ; \mathrm{LB}=1.88 \mathrm{~mm} ; \mathrm{LE} / \mathrm{LP}=2.34 ; \mathrm{WE} / \mathrm{WP}=$ $1.32 ; \mathrm{WP} / \mathrm{LP}=1.29 ; \mathrm{LE} / \mathrm{LAED}=1.66 \pm 0.07 ;$ $\mathrm{LAN} /(\mathrm{LE}+\mathrm{LP})=0.85$. $+(\mathrm{n}=8$; mean and standard deviation): $\mathrm{LE}=1.39 \pm 0.03 \mathrm{~mm} ; \mathrm{WE}=1.01 \pm 0.05 \mathrm{~mm}$; $\mathrm{LP}=0.53 \pm 0.03 \mathrm{~mm} ; \mathrm{WP}=0.71 \pm 0.02 \mathrm{~mm} ; \mathrm{LAN}=$ $1.63 \pm 0.05 \mathrm{~mm} ; \mathrm{LSP}=0.30 \pm 0.02 \mathrm{~mm} ; \mathrm{LB}=2.24 \pm$ $0.10 \mathrm{~mm} ; \mathrm{LE} / \mathrm{LP}=2.62 \pm 0.12 ; \mathrm{WE} / \mathrm{WP}=1.41 \pm 0.07$; $\mathrm{WP} / \mathrm{LP}=1.34 \pm 0.06 ;$ LE/LSP $=4.70 \pm 0.29 ;$ $\mathrm{LAN} /(\mathrm{LE}+\mathrm{LP})=0.85 \pm 0.02$.

Females slightly bigger than males, without enlarged first anterior and middle tarsomeres and apically darker hind femora. Spermatheca (Figs 45a-b) with sub- reniform basal part; distal part clearly developed, well distinct from basal part; duct moderately elongate, subbasally inserted, slightly robust, uncoiled, undulate or with a narrow coil.

Etymology. This new species is named after its collector E. Grobbelaar (SANC), in recognition of her kind and valuable collaboration.

Distribution. Kwazulu-Natal (Republic of South Africa) (Fig. 56). Southern-Eastern Afrotropical chorotype (cf. Biondi \& D'Alessandro, 2006).

Host plants. Heliotropium sp. (Boraginaceae).

\section{Longitarsus hexrivierbergensis sp.n.}

Type material. Holotype $\hat{\sigma}$, Republic of South Africa, Western Cape Province, $35 \mathrm{~km} \mathrm{NE}$ Ceres, 33ำ14.943'S, 19³8.435'E, 900 m, 15.x.2005 (karoo), on Echium plantagineum, P. Audisio, M. Biondi \& A. De Biase leg. (SANC). Paratypes: same locality, date, collectors and host plant as the holotype, 30,4 ㅇ (BAQ, SANC).

Diagnosis. This new species is externally very similar to L. debiasei sp.n. from which it differs in leg and antennal colour, slightly darker, with clearly obscured first antennomere in $L$. hexrivierbergensis, and by the shape of elytral apex, obliquely sub-truncate in $L$. hexrivierbergensis. In this new species the median lobe of aedeagus (Fig. 21) is characteristic in having the apical part sub-rounded with an inconspicuous small median tooth; the spermatheca of this new species is similar to that of L. sudafricanus sp.n. (Figs 33a-c, 35a-b).

Description. Holotype ( $\delta$ ) dorsal integument black, with evident metallic reflection. Body (Fig. 6) elongateoval $(\mathrm{LB}=1.90 \mathrm{~mm})$, moderately convex. Maximum pronotal width in middle: $0.66 \mathrm{~mm}$; maximum elytral width in middle: $0.98 \mathrm{~mm}$.

Head with rather impunctate vertex and frons, but with some small punctures impressed near frontal grooves; surface wrinkled. Frontal tubercles distinct, elongate, oblique, with smooth surface; frontal grooves finely impressed, distally divergent from ocular margin; frontal carina moderately wide, weakly raised; labrum subtrapezoidal, distally broadly rounded, clearly blackened; palpi strongly darkened; eyes sub-elliptical, normal size; antennae with partially but clearly darkened segment 1 , reddish segments $2-3$, partially darkened segment 4 , and entirely darkened segments 5-11; length of each segment of right antenna proportional to numerical sequence 24 : $15: 12: 15: 18: 14: 18: 18: 19: 19: 24(1=0.032$ $\mathrm{mm})$.

Pronotum scarcely transverse $(\mathrm{LP}=0.50 \mathrm{~mm}$; WP/LP $=$ 1.33 ), laterally very slightly rounded, basally slightly narrower; lateral and basal margin very finely bordered; punctation dense and evenly distributed with clearly impressed and rather little punctures on microreticulate surface. Scutellum wide, sub-triangular, with weakly reticulate surface.

Elytra elongate $(\mathrm{LE}=1.38 \mathrm{~mm} ; \mathrm{LE} / \mathrm{LP}=2.75)$, laterally rounded, apically obliquely sub-truncate; pygidium partially uncovered; punctation similar to that on pronotum but with larger punctures impressed on a smooth surface; humeral calli absent; subapterous wings. 

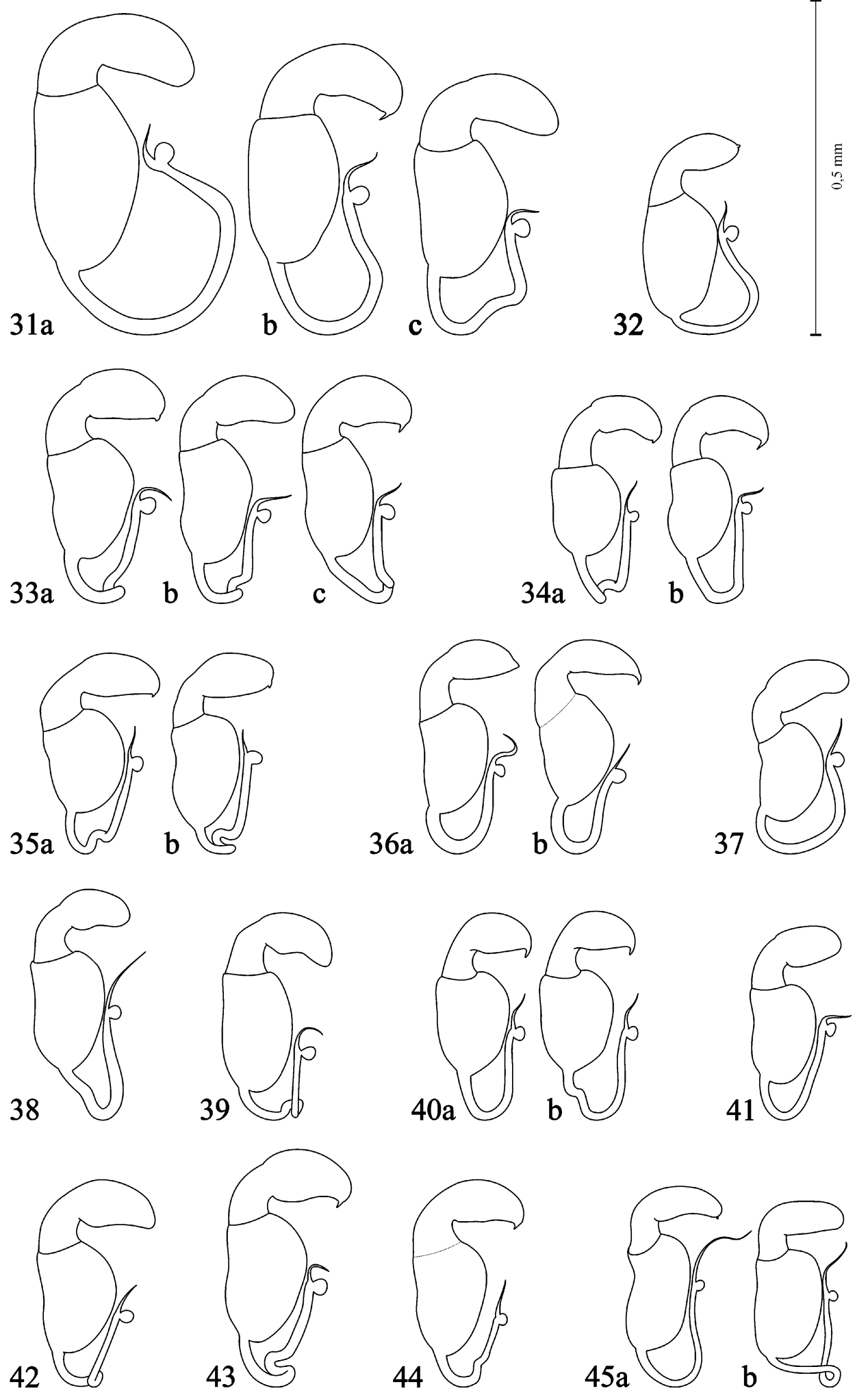

Figs 31-45. Spermatheca in Longitarsus capensis species-group: L. afromeridionalis sp.n., Western Cape Province, Road Quoin Point-Cape Agulhas (31a, 31b, 31c); L. rouxi sp.n., Western Cape Province, Cedarberg (32); L. sudafricanus sp.n., Western Cape Province, Piketberg (33a); L. sudafricanus sp.n., Western Cape Province, road Citrusdal-Middelberg Pass (33b, 33c); L. capensis Baly, Western Cape Province, Du Toitskloof Pass (34a, 34b); L. hexrivierbergensis sp.n., Western Cape Province, 35 km NE Ceres (35a, 35b); L. debiasei sp.n., Western Cape Province, Kogmanskloof (36a, 36b); L. lugubris Biondi, Western Cape Province, neigh. Pakhuis Pass (37); L. malherbei sp.n., Western Cape Province, Algeria, Cederberg Nature Reserve (38); L. melanicus Biondi, Western Cape Province, Danabaai (39); L. transvaalensis Biondi, Eastern Transvaal, Nelshoogte Pass (40a, 40b); L. cedarbergensis Biondi, Western Cape Province, road Clanwilliam-Pakhuis Pass (41); L. luctuosus Biondi, Western Cape Province, between Akkedisberg Pass and Stanford (42); L. piketbergensis sp.n., Western Cape Province, Piketberg (43); L. neseri Biondi, Western Cape Province, Piketberg (44); L. grobbelaariae sp.n., Kwazulu-Natal, Vryheid Hill Nature Reserve (45a, 45b). 

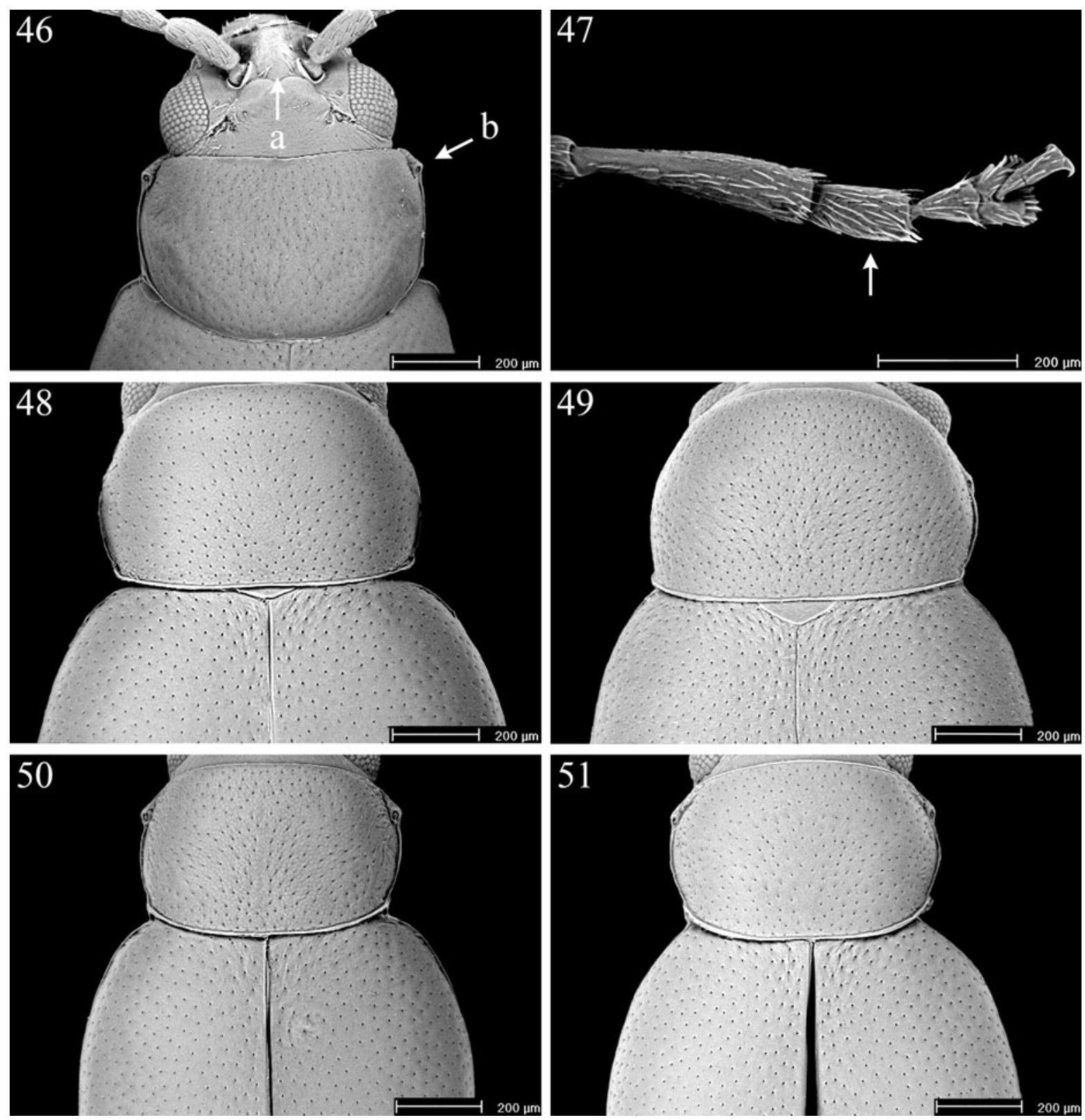

Figs 46-51. Scanning electron micrographs: frontal carina (a) and anterior angles of pronotum (b) of L. grobbelaariae sp.n. (46); first front tarsomere of Longitarsus melanicus Biondi (47); pronotum and basal part of elytra of Longitarsus afromeridionalis sp.n. (48); ditto of L. melanicus Biondi (49); ditto of L. piketbergensis sp.n. (50); ditto of L. sudafricanus sp.n. (51).

Legs with reddish tibiae and tarsi; ungual segment very weakly darkened; anterior and middle femora basally slightly darkened; hind femora black; from basal third hind tibiae externally curved and from basal fourth dentate on external edge; apical spur of hind tibiae long about $4 / 5$ of width of tibial apex, reddish; first anterior and middle tarsomeres slightly dilated.

Ventral part entirely black; last sternite without dimples or special preapical impressions.

Median lobe of aedeagus (Fig. 21) elongate (LAED = $0.95 \mathrm{~mm}$; LE/LAED = 1.45), slender, in ventral view weakly narrower in middle; apical part sub-rounded, with very wide, flattened and scarcely evident median small tooth; ventral sulcus elongate, moderately wide, basally not interrupted; apical half of dorsal sulcus well visible, deeply impressed; dorsal ligula very narrow; in lateral view basal third of median lobe weakly curved and then straight with apex clearly bent in ventral direction.

Variation. $\hat{\delta}(\mathrm{n}=4$; mean and standard deviation): LE $=1.30 \pm 0.09 \mathrm{~mm} ; \mathrm{WE}=0.92 \pm 0.08 \mathrm{~mm} ; \mathrm{LP}=0.48 \pm$ $0.04 \mathrm{~mm} ; \mathrm{WP}=0.63 \pm 0.04 \mathrm{~mm} ; \mathrm{LAN}=1.43 \pm 0.16 \mathrm{~mm}$; $\mathrm{LAED}=0.92 \pm 0.05 \mathrm{~mm} ; \mathrm{LB}=1.88 \pm 0.12 \mathrm{~mm} ; \mathrm{LE} / \mathrm{LP}=$ $2.74 \pm 0.04 ; \mathrm{WE} / \mathrm{WP}=1.47 \pm 0.08 ; \mathrm{WP} / \mathrm{LP}=1.32 \pm$ $0.05 ; \mathrm{LE} / \mathrm{LAED}=1.41 \pm 0.03 ; \mathrm{LAN} /(\mathrm{LE}+\mathrm{LP})=0.80 \pm$ 0.04 . 9 ( $\mathrm{n}=4$; mean and standard deviation): $\mathrm{LE}=1.55$ $\pm 0.15 \mathrm{~mm} ; \mathrm{WE}=1.12 \pm 0.10 \mathrm{~mm} ; \mathrm{LP}=0.54 \pm 0.04 \mathrm{~mm}$; $\mathrm{WP}=0.73 \pm 0.06 \mathrm{~mm} ; \mathrm{LAN}=1.45 \pm 0.13 \mathrm{~mm} ; \mathrm{LSP}=$ $0.30 \pm 0.02 \mathrm{~mm} ; \mathrm{LB}=2.37 \pm 0.22 \mathrm{~mm} ; \mathrm{LE} / \mathrm{LP}=2.87 \pm$ $0.06 ; \mathrm{WE} / \mathrm{WP}=1.54 \pm 0.02 ; \mathrm{WP} / \mathrm{LP}=1.34 \pm 0.02 ;$ $\mathrm{LE} / \mathrm{LSP}=5.18 \pm 0.49 ; \mathrm{LAN} /(\mathrm{LE}+\mathrm{LP})=0.69 \pm 0.01$. 

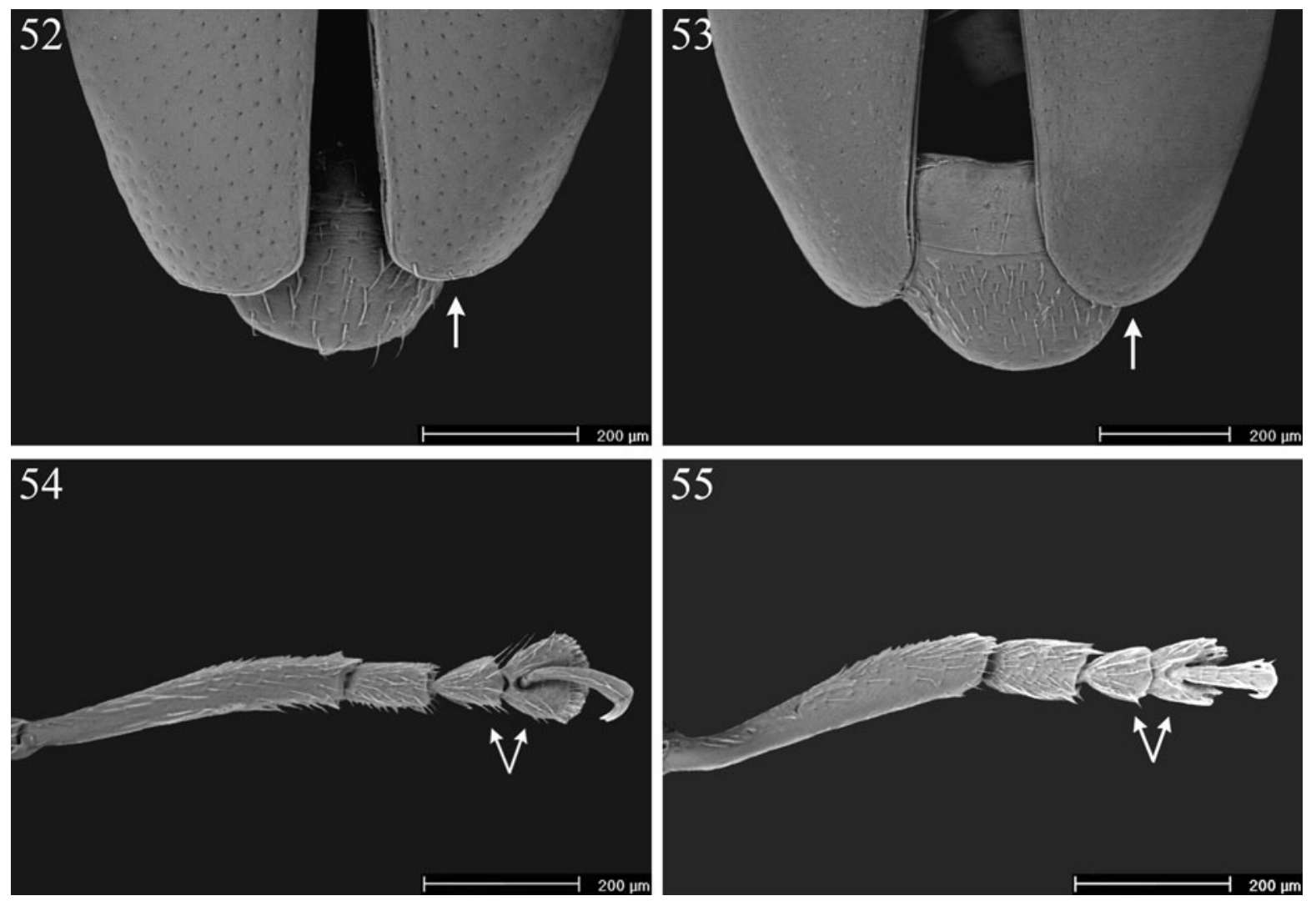

Figs 52-55. Scanning electron micrographs: elytral apex of Longitarsus malherbei sp.n. (52); ditto of L. transvaalensis Biondi (53); second and third front tarsomeres of Longitarsus cedarbergensis Biondi (54); ditto of L. neseri Biondi (55).

Paratypes similar in shape, sculpture and colour to the holotype, but often with straight not curved hind tibiae, and more strongly darkened front and middle femora. Females generally bigger than males, with shorter antennae, flatter frontal carina and first anterior and middle tarsomeres not enlarged. Spermatheca (Figs 35a-b) with sub-reniform, sometimes sub-globose, basal part; distal part well developed, clearly distinct from basal part; duct moderately elongate, rather thin, basally or sub-basally inserted, with one complete or incomplete coil.

Etymology. This new species is named after Hexrivierberg Mountains (Republic of South Africa, Western Cape Province).

Distribution. Hexrivierberg area (Republic of South Africa: Western Cape Province) (Fig. 57). Southern-Western Afrotropical chorotype (cf. Biondi \& D'Alessandro, 2006).

Host plants. Echium plantagineum (Boraginaceae).

\section{Longitarsus luctuosus Biondi}

Longitarsus luctuosus Biondi, 1999: 520.

Material examined. Republic of South Africa: Western Cape Province, between Akkedisberg Pass and Stanford (R326)

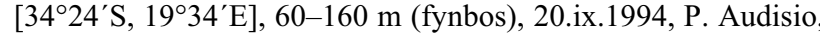
M. Biondi \& M. Bologna leg., 1 đ (holotype) (BAQ); ditto, 50 , 9 (paratypes) (BAQ); Western Cape Province, Akkedisbergpas, neigh. Stanford, $34^{\circ} 26.534^{\prime} \mathrm{S}, 1^{\circ} 28.362^{\prime} \mathrm{E}, 30 \mathrm{~m}$ 21.ix.2006 (fynbos), on Echium plantagineum, M. Biondi \& A. De Biase leg., 30, 6 ㅇ (BAQ); Western Cape Province, NNW of Stellenbosch, $33^{\circ} 50^{\prime} \mathrm{S}, 18^{\circ} 46^{\prime} \mathrm{E}$, 13.vii.1995, on Echium plantagineum (leaves with holes), S. Neser leg., 15 0 , 20 우 (with eggs) (paratypes) (SANC, BAQ).
Distribution. Southern-western part of the Western Cape Province (Republic of South Africa) (Fig. 57). SouthernWestern Afrotropical chorotype (cf. Biondi \& D'Alessandro, 2006).

Host plants. Echium plantagineum (Boraginaceae).

\section{Longitarsus lugubris Biondi}

Longitarsus lugubris Biondi, 1999: 522.

Material examined. Republic of South Africa: Northern Cape Province, 3-8 km SW of Nieuwoudtville [31 $24^{\prime} \mathrm{S}$, $29^{\circ} 04^{\prime} \mathrm{E}$ ], 700-760 m, 15.ix.1994 (fynbos and grassland), P. Audisio, M. Biondi \& M. Bologna leg., $10^{\widehat{t}}$ (holotype) (BAQ); ditto, 7ठ, $9+$ (paratypes) (BAQ, BMNH); Western Cape Province, road Clanwilliam-Pakhuis Pass $\left[32^{\circ} 08^{\prime} \mathrm{S}, 1^{\circ} 56^{\prime} \mathrm{E}\right]$, 200-900 m, 11.ix.1994 (fynbos and xeric grassland), P. Audisio, M. Biondi \& M. Bologna leg., 10ðิ, $8 \uparrow$ (BAQ, SANC); Western Cape Province, Pakhuis Pass, 883 m, 3208.898'S, $19^{\circ} 01.993^{\prime} \mathrm{E}, 14.1 x .2006$ (fynbos), on Lobostemon cf. dorotheae and L. cf. trichotomus, M. Biondi \& A. De Biase leg., 40ิ, 1 ㅇ (BAQ); Western Cape Province, neigh. Pakhuis Pass, $666 \mathrm{~m}$, $32^{\circ} 08.128^{\prime} \mathrm{S}, 18^{\circ} 57.568^{\prime} \mathrm{E}, 14.1 \mathrm{x} .2006$ (fynbos), on Lobostemon cf. dorotheae and $L$. cf. trichotomus, M. Biondi \& A. De Biase leg., 10 , 4 ? (BAQ).

Distribution. Bokkeveldeberg and Cedarberg areas (Republic of South Africa: Northern and Western Cape Province) (Fig. 57). Southern-Western Afrotropical chorotype (cf. Biondi \& D'Alessandro, 2006).

Host plants. Lobostemon cf. dorotheae and L. cf. trichotomus (Thumb.) DC. (Boraginaceae).

\section{Longitarsus malherbei sp.n.}

Type material. Holotype $\delta$, Republic of South Africa, Western Cape Province, Pakhuis Pass, 883 m, 14.ix.2006 (fyn- 


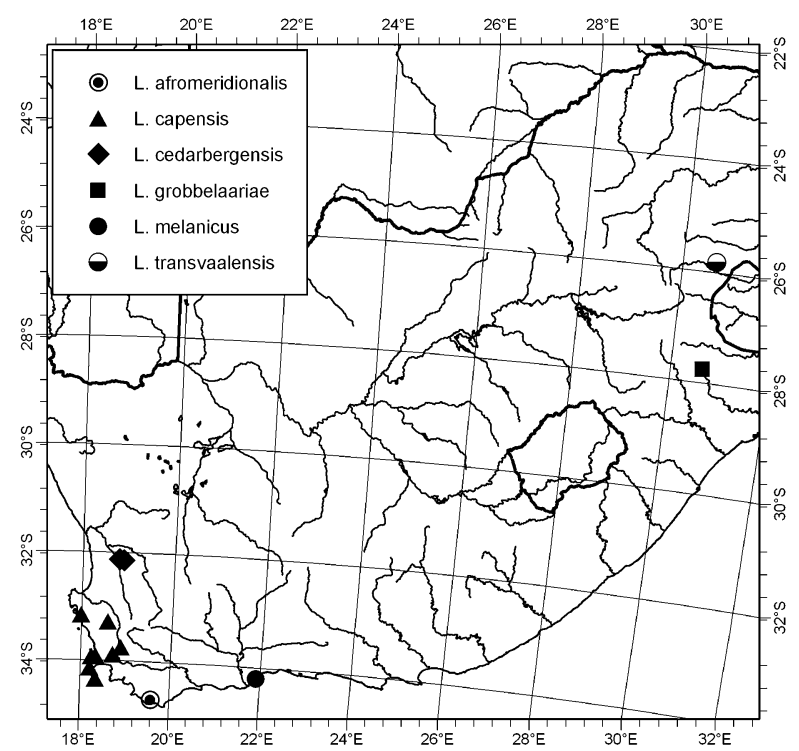

Fig. 56. Distribution of: Longitarsus afromeridionalis sp.n., L. capensis Baly, L. cedarbergensis Biondi, L. grobbelaariae sp.n., L. melanicus Biondi and L. transvaalensis Biondi.

bos), $32^{\circ} 08.898^{\prime} \mathrm{S}, 19^{\circ} 01.993^{\prime} \mathrm{E}$, on Lobostemon cf. dorotheae, M. Biondi \& A. De Biase leg. (SANC). Paratypes: Republic of South Africa, Western Cape Province, Algeria, Cederberg Nature Reserve, $32^{\circ} 22.702^{\prime} \mathrm{S}, 1^{\circ} 03.879^{\prime} \mathrm{E}, 541 \mathrm{~m}, 14.1 x .2006$ (fynbos), on Lobostemon cf. dorotheae and L. cf. trichotomus, M. Biondi \& A. De Biase leg., 13 ơ, 13 ? (BAQ, SANC).

Diagnosis. In its small size $(\mathrm{LB}=1.90 \mathrm{~mm}$ in males and $=2.53 \mathrm{~mm}$ in females) and long antennae $[\mathrm{LAN} /(\mathrm{LE}+\mathrm{LP})=1.05$ in males and $=0.96$ in females $]$ this new species is similar to L. transvaalensis, from which it differs externally in the colour of front and middle femora, yellowish in L. malherbei sp.n., and shape of the elytral apex, obliquely sub-truncate in L. malherbei sp.n. (Figs 52-53). The two species also are easily distinguishable by the median lobe of aedeagus, which has a basally interrupted ventral sulcus in $L$. malherbei sp.n. (Figs 18, 27), and a different shaped spermatheca, with the more elongate basal part and proximal tract sinuous in L. malherbei sp.n. (Figs 38, 40a-b).

Description. Holotype ( $\delta$ dorsal integument black, with evident bronze metallic reflection. Body (Fig. 9) elongate-elliptical ( $\mathrm{LB}=1.90 \mathrm{~mm}$ ), slightly convex. Maximum pronotal width in middle: $0.58 \mathrm{~mm}$; maximum elytral width in middle: $0.80 \mathrm{~mm}$.

Head with rather impunctate vertex and frons, but with some coarse and large punctures near frontal grooves; surface shagreened. Frontal tubercles scarcely delimited, moderately elongate, oblique, with smooth surface; frontal grooves finely impressed, distally divergent from ocular margin; frontal carina little wide, clearly raised; labrum sub-trapezoidal, blackened; palpi strongly darkened; eyes sub-elliptical, normal size; antennae with yellowish segments 1-4 and gradually darkened segments 5-11; length of each segment of right antenna proportional to numerical sequence $22: 14: 12: 16: 19: 18$ : $22: 21: 21: 21: 25(1=0.032 \mathrm{~mm})$.

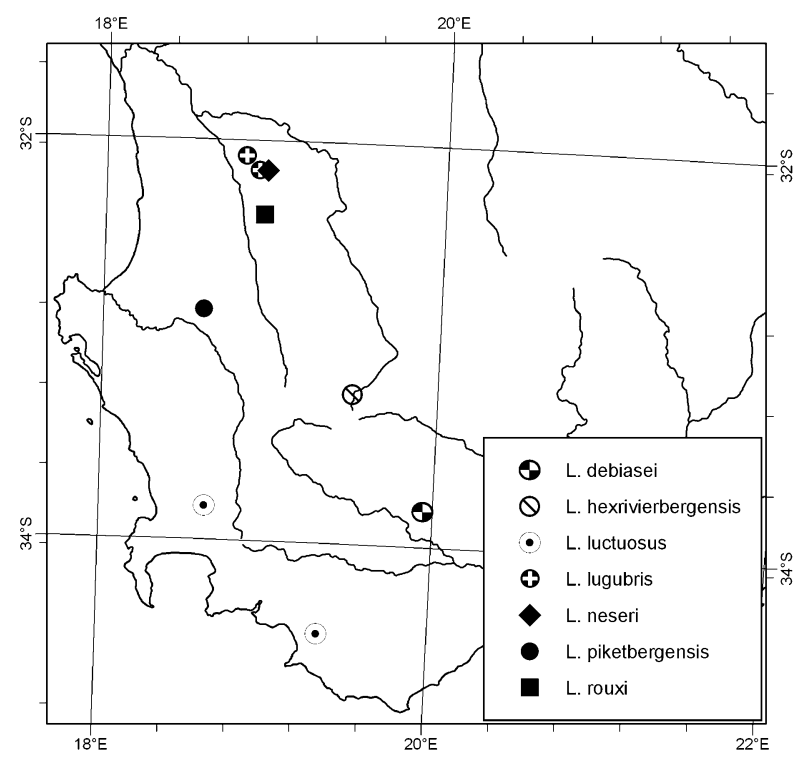

Fig. 57. Distribution of: Longitarsus debiasei sp.n., L. hexrivierbergensis sp.n., L. luctuosus Biondi, L. lugubris Biondi, L. neseri Biondi, L. piketbergensis sp.n. and L. rouxi n.sp.

Pronotum sub-rectangular $(\mathrm{LP}=0.40 \mathrm{~mm} ; \mathrm{WP} / \mathrm{LP}=$ 1.44) laterally slightly rounded, basally slightly narrower; lateral margin weakly bordered and basal one very finely bordered; punctation uniformly distributed with rather clearly and densely impressed minute punctures on microreticulate surface. Scutellum moderately wide, semi-circular, with finely wrinkled surface.

Elytra elongate $(\mathrm{LE}=1.10 \mathrm{~mm}$; $\mathrm{LE} / \mathrm{LP}=2.75)$, laterally rounded, apically obliquely sub-truncate; pygidium uncovered; punctation similar to that on pronotum but impressed on a very smooth surface; humeral calli absent; subapterous wings.

Legs with yellowish tibiae, tarsi, anterior and middle femora; hind femora dorsally slightly darkened; from basal fourth hind tibiae dentate on external edge; apical spur of hind tibiae long about $1 / 2$ of tibial apex width, reddish; first anterior and middle tarsomeres slightly dilated.

Ventral part entirely black; last sternite without dimples or special preapical impressions.

Median lobe of aedeagus (Fig. 27) moderately elongate $(\mathrm{LAED}=0.75 \mathrm{~mm} ; \mathrm{LE} / \mathrm{LAED}=1.47)$, slightly slender, in ventral view medially very slightly narrower; apical part sub-triangular, with an inconspicuous small median tooth; ventral sulcus very wide, basally interrupted; dorsal sulcus V-shaped, apical third very visible; dorsal ligula very narrow; in lateral view basal third of median lobe evenly curved and then straight with apex slightly bent in ventral direction.

Variation. $\hat{\sigma}(\mathrm{n}=10$; mean and standard deviation): $\mathrm{LE}=1.04 \pm 0.07 \mathrm{~mm} ; \mathrm{WE}=0.77 \pm 0.05 \mathrm{~mm} ; \mathrm{LP}=0.39$ $\pm 0.03 \mathrm{~mm} ; \mathrm{WP}=0.55 \pm 0.04 \mathrm{~mm} ; \mathrm{LAN}=1.55 \pm 0.10$ $\mathrm{mm} ; \mathrm{LAED}=0.77 \pm 0.02 \mathrm{~mm} ; \mathrm{LB}=1.75 \pm 0.12 \mathrm{~mm}$; $\mathrm{LE} / \mathrm{LP}=2.69 \pm 0.07 ; \mathrm{WE} / \mathrm{WP}=1.39 \pm 0.04 ; \mathrm{WP} / \mathrm{LP}=$ $1.43 \pm 0.03 ; \mathrm{LE} / \mathrm{LAED}=1.35 \pm 0.08 ; \mathrm{LAN} /(\mathrm{LE}+\mathrm{LP})=$ $1.09 \pm 0.04$. ㅇ $(\mathrm{n}=10$; mean and standard deviation $)$ : LE 


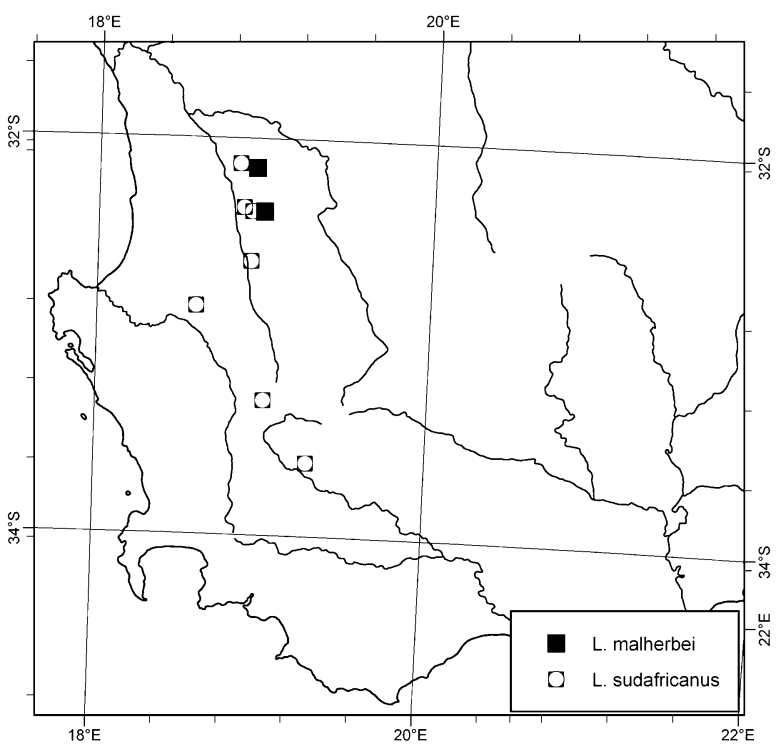

Fig. 58. Distribution of: Longitarsus malherbei sp.n. and $L$. sudafricanus sp.n.

$=1.31 \pm 0.12 \mathrm{~mm} ; \mathrm{WE}=0.99 \pm 0.08 \mathrm{~mm} ; \mathrm{LP}=0.45 \pm$ $0.04 \mathrm{~mm} ; \mathrm{WP}=0.65 \pm 0.05 \mathrm{~mm} ; \mathrm{LAN}=1.72 \pm 0.13 \mathrm{~mm}$; $\mathrm{LSP}=0.34 \pm 0.02 \mathrm{~mm} ; \mathrm{LB}=2.20 \pm 0.18 \mathrm{~mm} ; \mathrm{LE} / \mathrm{LP}=$ $2.89 \pm 0.10 ; \mathrm{WE} / \mathrm{WP}=1.53 \pm 0.03 ; \mathrm{WP} / \mathrm{LP}=1.44 \pm$ $0.03 ; \mathrm{LE} / \mathrm{LSP}=3.90 \pm 0.28 ; \mathrm{LAN} /(\mathrm{LE}+\mathrm{LP})=0.98 \pm$ 0.02 .

Paratypes very similar in shape, sculpture and colour to holotype, but sometimes with darkened distal tarsomeres. Females bigger than males, with shorter antennae, flatter frontal carina and first anterior and middle tarsomeres not enlarged. Spermatheca (Fig. 38) with sub-reniform basal part; distal part moderately developed, clearly distinct from basal part; duct moderately elongate, rather robust, basally inserted, uncoiled, narrowly arcuate, and proximal tract generally sinuous.

Etymology. The new species is named after D. Malherbe (Republic of South Africa, Cedarberg Wilderness Nature Reserve, Algeria) for his kind assistance during our visit of the Nature Reserve.

Distribution. Cedarberg area (Republic of South Africa: Western Cape Province) (Fig. 58). Southern-Western Afrotropical chorotype (cf. Biondi \& D'Alessandro, 2006).

Host plants. Lobostemon cf. dorotheae and L. cf. trichotomus (Boraginaceae).

\section{Longitarsus melanicus Biondi}

Longitarsus melanicus Biondi, 1999: 523.

Material examined. Republic of South Africa: Western Cape Province, Danabaai, $6 \mathrm{~km} \mathrm{~W}$ of Mossel Bay, 34²12.067'S, $22^{\circ} 02.973^{\prime} \mathrm{E}, 0-60 \mathrm{~m}$ (coastal fynbos), 19.ix.1994, on Lobostemon sp., P. Audisio, M. Biondi \& M. Bologna leg., 10 (holotype) (BAQ); ditto, 79 (with eggs) (paratypes) (BAQ); ditto, 120 m, 19.ix.2006, on Lobostemon cf. marlothii, M. Biondi \& A. De Biase leg., 7 $\delta, 7$ (some specimens mating) (BAQ).

Distribution. Southern coastal area of the Western Cape Province (Republic of South Africa) (Fig. 56). SouthernWestern Afrotropical chorotype (cf. Biondi \& D'Alessandro, 2006).

Host plants. Lobostemon cf. marlothii Levyns (Boraginaceae).

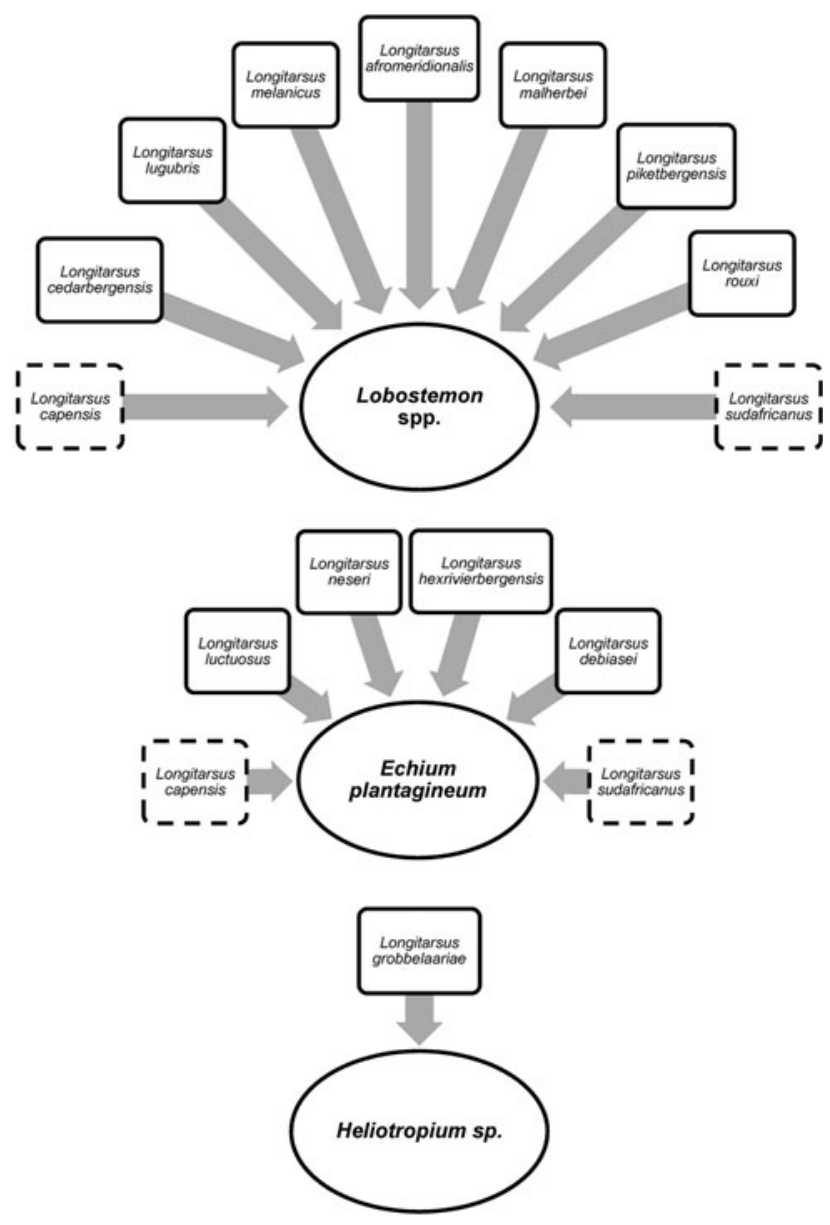

Fig. 59. Host-plants of the Longitarsus capensis speciesgroup.

\section{Longitarsus neseri Biondi}

Longitarsus neseri Biondi, 1999: 525.

Material examined. Republic of South Africa: Western Cape Province, Piketberg, $12^{\circ} 44^{\prime} \mathrm{S}, 2^{\circ} 25^{\prime} \mathrm{E}$ (sic!) [correct coordinates of Piketberg: $\left.32^{\circ} 54^{\prime} \mathrm{S}, 18^{\circ} 44^{\prime} \mathrm{E}\right]$, 8.vii.1986, S. Neser leg.,

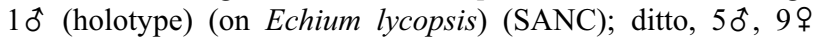
(with eggs) (paratypes) (SANC; BAQ).

Distribution. Piketberg area (Republic of South Africa: Western Cape Province) (Fig. 57). Southern-Western Afrotropical element (cf. Biondi \& D'Alessandro, 2006).

Host plants. Echium plantagineum (= lycopsis auct.) (Boraginaceae).

\section{Longitarsus piketbergensis sp.n.}

Type material. Holotype $\hat{\delta}$, Republic of South Africa, Western Cape Province, Piketberg, 32 $51.408^{\prime} \mathrm{S}, 18^{\circ} 44.176^{\prime} \mathrm{E}$, 591 m, 17.ix.2006, (fynbos), Lobostemon fruticosus, M. Biondi \& A. De Biase leg. (SANC). Paratypes: same locality, date, host plant and collectors of the holotype, $40,4 \%$ (BAQ, SANC).

Diagnosis. On the basis of the median lobe of the aedeagus and spermatheca, this new species shows close affinities mainly with $L$. sudafricanus sp.n. (Figs 23, 26, $33 \mathrm{a}-\mathrm{c}, 43)$, from which it is easy distinguishable by having a median lobe that is more slender in ventral view and more strongly arcuate in lateral view.

Description. Holotype ( $₫$ ) dorsal integument black, with evident metallic reflection. Body (Fig. 12) elongate- 
oval $(\mathrm{LB}=2.13 \mathrm{~mm})$, moderately convex. Maximum pronotal width in middle: $0.73 \mathrm{~mm}$; maximum elytral width in middle: $1.09 \mathrm{~mm}$.

Head with impunctate vertex and frons, but with some coarse and large punctures impressed near frontal grooves; surface finely wrinkled and sparsely punctulated between eyes. Frontal tubercles finely delimited, slightly elongate, oblique, with smooth surface; frontal grooves finely impressed, distally divergent from ocular margin; frontal carina moderately wide, weakly raised; labrum sub-trapezoidal, blackened; palpi strongly darkened; eyes sub-elliptical, normal size; antennae with partially darkened segment 1 , yellowish segments $2-3$, and darkened segments 4-11; length of each segment of right antenna proportional to numerical sequence $25: 15: 13: 18: 22$ : $20: 20: 20: 20: 22: 28(1=0.032 \mathrm{~mm})$.

Pronotum transverse $(\mathrm{WP}=0.50 \mathrm{~mm} ; \mathrm{WP} / \mathrm{LP}=1.45)$ laterally moderately rounded; lateral and basal margin finely bordered; punctuation uniformly distributed with rather little and moderately but densely impressed punctures on a microreticulate surface. Scutellum wide, semicircular, with smooth surface.

Elytra elongate $(\mathrm{LE}=1.43 \mathrm{~mm} ; \mathrm{LE} / \mathrm{LP}=2.85)$, laterally rounded, apically sub-truncate; pygidium partially uncovered; punctation similar to that on pronotum but impressed on a very smooth surface; humeral calli absent; subapterous wings.

Legs with yellowish tibiae, tarsi, anterior and middle femora; third and fifth tarsomeres slightly darkened; hind femora partially blackened; from basal third hind tibiae dentate on external edge; apical spur long about 3/5 of width of tibial apex, reddish; first anterior and middle tarsomeres very weakly dilated.

Ventral part entirely black; last sternite without dimples or special preapical impressions.

Median lobe of aedeagus (Fig. 26) clearly elongate $(\mathrm{LAED}=0.86 \mathrm{~mm}$; LE/LAED $=1.65)$, slender; in ventral view medially weakly narrower; apical part lanceolate, with an evident median small tooth, apically sub-rounded; ventral sulcus elongate, wider in apical fourth, then narrower, basally not interrupted; dorsal sulcus V-shaped and clearly visible in apical fourth; dorsal ligula very narrow; in lateral view basal half of median lobe regularly curved and then rather straight with apical part distinctly sinuous.

Variation. ô $(\mathrm{n}=5$; mean and standard deviation $)$ : LE $=1.29 \pm 0.13 \mathrm{~mm} ; \mathrm{WE}=0.98 \pm 0.09 \mathrm{~mm} ; \mathrm{LP}=0.47 \pm$ $0.03 \mathrm{~mm} ; \mathrm{WP}=0.65 \pm 0.06 \mathrm{~mm} ; \mathrm{LAN}=1.54 \pm 0.16 \mathrm{~mm}$; $\mathrm{LAED}=0.85 \pm 0.03 \mathrm{~mm} ; \mathrm{LB}=1.96 \pm 0.23 \mathrm{~mm} ; \mathrm{LE} / \mathrm{LP}=$ $2.73 \pm 0.09 ; \mathrm{WE} / \mathrm{WP}=1.51 \pm 0.02 ; \mathrm{WP} / \mathrm{LP}=1.37 \pm$ $0.05 ; \mathrm{LE} / \mathrm{LAED}=1.51 \pm 0.11 ; \mathrm{LAN} /(\mathrm{LE}+\mathrm{LP})=0.88 \pm$ 0.03 . $~ \&(\mathrm{n}=4$; mean and standard deviation $): \mathrm{LE}=1.63$ $\pm 0.15 \mathrm{~mm} ; \mathrm{WE}=1.27 \pm 0.13 \mathrm{~mm} ; \mathrm{LP}=0.56 \pm 0.06 \mathrm{~mm}$; $\mathrm{WP}=0.75 \pm 0.06 \mathrm{~mm} ; \mathrm{LAN}=1.70 \pm 0.20 \mathrm{~mm} ; \mathrm{LSP}=$ $0.34 \pm 0.02 \mathrm{~mm} ; \mathrm{LB}=2.58 \pm 0.42 \mathrm{~mm} ; \mathrm{LE} / \mathrm{LP}=2.91 \pm$ $0.08 ; \mathrm{WE} / \mathrm{WP}=1.68 \pm 0.07 ; \mathrm{WP} / \mathrm{LP}=1.34 \pm 0.06$; $\mathrm{LE} / \mathrm{LSP}=4.84 \pm 0.37 ; \mathrm{LAN} /(\mathrm{LE}+\mathrm{LP})=0.77 \pm 0.03$.

Paratypes very similar in shape, sculpture and colour to the holotype. Median lobe of aedeagus in some males more strongly narrowed. Females larger than males, with shorter antennae and first anterior and middle tarsomeres not enlarged. Spermatheca (Fig. 43) with sub-reniform basal part; distal part clearly developed, distinct from basal part; duct moderately elongate, sub-basally inserted, with two narrow coils.

Etymology. This new species is named after Piketberg Mountains (Republic of South Africa, Western Cape Province).

Distribution. Piketberg area (Republic of South Africa: Western Cape Province) (Fig. 57). Southern-Western Afrotropical chorotype (cf. Biondi \& D'Alessandro, 2006).

Host plants. Lobostemon fruticosus (Boraginaceae).

\section{Longitarsus rouxi sp.n.}

Type material. Holotype $\delta$, Republic of South Africa, Western Cape Province, Cedarberg, above Algeria Forest Station, 32 $22^{\prime}$ S, $19^{\circ} 04^{\prime}$ E, 22.iv.1992, C. Roux leg. (SANC). Paratypes: same locality, date and collectors as the holotype, 1 o (SANC); Republic of South Africa, Western Cape Province, Algeria, Cedarberg Nature Reserve, $32^{\circ} 22.702 \mathrm{~S}, 19^{\circ} 03.879^{\prime} \mathrm{E}$, 541 m, 14.ix.2006 (fynbos), on Lobostemon sp., M. Biondi \& A. De Biase leg., 1 ( $\mathrm{BAQ})$.

Diagnosis. This new species is externally similar to the other species of the capensis group, but because of the peculiar shape of the median lobe of its aedeagus and of the spermatheca (Figs 22, 32) it occupies a very isolated taxonomical position.

Description. Holotype ( $₫)$ not a mature specimen. Dorsal integument tending to black, with evident greenish metallic reflection. Body (Fig. 13) elongate-elliptical (LB $=1.73 \mathrm{~mm}$ ), moderately convex. Maximum pronotal width in middle: $0.58 \mathrm{~mm}$; maximum elytral width in middle: $0.75 \mathrm{~mm}$.

Head with impunctate vertex and frons, but laterally with some moderately impressed large punctures; surface very slightly wrinkled. Frontal tubercles finely delimited, moderately elongate, oblique, with smooth surface; frontal grooves finely impressed, distally divergent from ocular margin; frontal carina slightly widened, moderately raised; labrum sub-trapezoidal, clearly blackened; palpi strongly darkened; eyes sub-elliptical, normal size; antennae with yellowish segments 1-4 and slightly obscured segments 5-11; length of each segment of right antenna proportional to numerical sequence $21: 12: 12$ : $16: 18: 16: 17: 18: 18: 20: 24(1=0.032 \mathrm{~mm})$.

Pronotum slightly transverse $(\mathrm{LP}=0.44 \mathrm{~mm}$; WP/LP $=$ 1.31 ), laterally moderately rounded, basally slightly narrower; lateral margin weakly bordered and basal one very finely bordered; punctation uniformly distributed with rather little but densely and clearly impressed punctures on weakly microreticulate surface. Scutellum wide, semicircular, with smooth surface.

Elytra elongate $(\mathrm{LE}=1.16 \mathrm{~mm} ; \mathrm{LE} / \mathrm{LP}=2.66)$, laterally rounded, apically sub-truncate; pygidium partially uncovered; punctation similar to that on pronotum, impressed on a very smooth surface; humeral calli absent; subapterous wings.

Legs with yellowish tibiae, tarsi, and anterior and middle femora; hind femora dorsally slightly darkened; distal three quarters of hind tibiae dentate on external 
edge; apical spur of hind tibiae long about $3 / 5$ of tibial apex width, reddish; first anterior and middle tarsomeres moderately dilated.

Ventral part tending to black; last three sternites with an evident preapical hollow with a more deeply impressed median longitudinal furrow.

Median lobe of aedeagus (Fig. 22) slightly elongated $(\mathrm{LAED}=0.81 \mathrm{~mm} ; \mathrm{LE} / \mathrm{LAED}=1.43)$, thickset, in ventral view sub-parallel; apical part obtusely rounded, with an evident small median tooth, apically sub-acute; apical third of ventral sulcus very wide then clearly convergent towards middle, basally strongly narrower, laterally subparallel; dorsal sulcus elongate, wide, well visible in apical third; in lateral view basal third of median lobe clearly bent and then straightens; apical fifth distinctly thinner than basal $4 / 5 \mathrm{~s}$; rather straight apex.

Variation. $\delta \hat{~}(\mathrm{n}=1$; immature specimen $): \mathrm{LE}=1.16$ $\mathrm{mm} ; \mathrm{WE}=0.75 \mathrm{~mm} ; \mathrm{LP}=0.44 \mathrm{~mm} ; \mathrm{WP}=0.58 \mathrm{~mm}$; $\mathrm{LAN}=1.60 \mathrm{~mm} ; \mathrm{LAED}=0.81 \mathrm{~mm} ; \mathrm{LB}=1.73 \mathrm{~mm}$ $\mathrm{LE} / \mathrm{LP}=2.66 ; \mathrm{WE} / \mathrm{WP}=1.30 ; \mathrm{WP} / \mathrm{LP}=1.31 ; \mathrm{LE} / \mathrm{LAED}$ $=1.43 ; \mathrm{LAN} /(\mathrm{LE}+\mathrm{LP})=1.00$. $~(\mathrm{n}=2 ;$ mean and standard deviation): $\mathrm{LE}=1.41 \pm 0.23 \mathrm{~mm} ; \mathrm{WE}=0.90 \pm 0.04$ $\mathrm{mm} ; \mathrm{LP}=0.48 \pm 0.06 \mathrm{~mm} ; \mathrm{WP}=0.66 \pm 0.08 \mathrm{~mm}$; LAN $=1.52 \pm 0.19 \mathrm{~mm} ; \mathrm{LSP}=0.30 \pm 0.01 \mathrm{~mm} ; \mathrm{LB}=2.09 \pm$ $0.11 \mathrm{~mm} ; \mathrm{LE} / \mathrm{LP}=2.93 \pm 0.10 ; \mathrm{WE} / \mathrm{WP}=1.38 \pm 0.11$; $\mathrm{WP} / \mathrm{LP}=1.36 \pm 0.01 ; \mathrm{LE} / \mathrm{LSP}=4.71 \pm 0.77$; $\mathrm{LAN} /(\mathrm{LE}+\mathrm{LP})=0.80 \pm 0.03$.

Females bigger than males, with shorter antennae, wider and flatter frontal carina and not enlarged first anterior and middle tarsomeres. The only completely mature female examined shows the following chromatic differences compared to the holotype: dorsal and ventral integument black, with weak greenish metallic reflection; antennae with almost entirely darkened segment 1 , slightly paler segments 2-4 and darkened segments 5-11; ungual segment slightly darkened; anterior and middle femora basally weakly darkened; hind femora clearly blackened. Spermatheca (Fig. 32) with sub-reniform basal part; distal part weakly developed, moderately distinct from basal part; duct elongate, basally inserted, uncoiled, widely arcuate, with very short descendent proximal tract.

Etymology. This new species is named after its first collector C. Roux (Republic of South Africa).

Distribution. Cedarberg area (Republic of South Africa: Western Cape Province) (Fig. 57). Southern-Western Afrotropical chorotype (cf. Biondi \& D'Alessandro, 2006).

Host plants. Lobostemon sp., probably $L$. cf. dorotheae or $L$. cf. trichotomus (Boraginaceae).

\section{Longitarsus sudafricanus sp.n.}

Type material. Holotype $\hat{\delta}$, Republic of South Africa, Western Cape Province, Piketberg, 32 $51.408^{\prime} \mathrm{S}, 18^{\circ} 44.176^{\prime} \mathrm{E}$, 591 m, 17.ix.2006, (fynbos), on Lobostemon fruticosus., M. Biondi \& A. De Biase leg. (SANC). Paratypes: same locality, date, host plant and collectors as the holotype, $7 \delta, 11$ ( $\mathrm{BAQ}$, SANC); Republic of South Africa, Western Cape Province, road Citrusdal-Middelberg Pass, 32³7.213'S, $1^{\circ} 03.324^{\prime} \mathrm{E}-$ $32^{\circ} 37.353^{\prime} \mathrm{S}, 1^{\circ} 08.142^{\prime} \mathrm{E}, 438-893 \mathrm{~m}, 13.1 x .2006$ (fynbos), on Lobostemon sp., M. Biondi \& A. De Biase leg., $13 \hat{\delta}$ and 23 ㅇ (BAQ); Western Cape Province, neigh. Pakhuis Pass, $32^{\circ} 08.128^{\prime} \mathrm{S}, 18^{\circ} 57.568^{\prime} \mathrm{E}, 666 \mathrm{~m}, 14 . i x .2006$ (fynbos), on
Lobostemon sp., M. Biondi \& A. De Biase leg., 10 (BAQ); Western Cape Province, Nieuwoudtspas nr. Algeria, $32^{\circ} 20.977^{\prime} \mathrm{S}, 1^{\circ} 00.410^{\prime} \mathrm{E}, 527 \mathrm{~m}, 14.1 x .2006$ (fynbos), on Lobostemon cf. dorotheae, M. Biondi \& A. De Biase leg., 4 우 (BAQ); Western Cape Province, Algeria, Cederberg Nature Reserve, $32^{\circ} 22.702^{\prime} \mathrm{S}, 1^{\circ} 03.879^{\prime} \mathrm{E}, 541 \mathrm{~m}, 14 . i x .2006$ (fynbos), on Lobostemon cf. dorotheae and L. cf. trichotomus, M. Biondi \& A. De Biase leg., 29 đે, 28 (BAQ); Western Cape

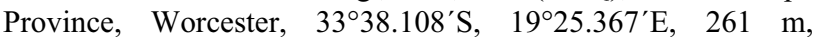
22.ix.2006 (road edge), on Echium plantagineum, M. Biondi \& A. De Biase leg., 30 , 18 ㅇ (BAQ); Western Cape Province, 4 $\mathrm{km}$ S Tulbagh, $33^{\circ} 19.387^{\prime} \mathrm{S}, 1^{\circ} 08.717^{\prime} \mathrm{E}, 171 \mathrm{~m}, 17 . \mathrm{ix} .2006$ (fynbos), on Echium plantagineum, M. Biondi \& A. De Biase leg., $60 \hat{0}, 17$ 우 (BAQ).

Diagnosis. This new species is very variable in some external morphological characters and the shape of the median lobe of the aedeagus and of the spermatheca. However, because of the shape of the median lobe of its aedeagus, L. sudafricanus sp.n. is considered to be closely related to L. afromeridionalis (Figs 23, 29), from which it differs mainly in the different shape of the spermatheca (Figs $31 \mathrm{a}-\mathrm{c}, 33 \mathrm{a}-\mathrm{c}$ ), which is similar that of $L$. hexrivierbergensis sp.n. and L. piketbergensis sp.n. (Figs 35a-b, 43).

Description. Holotype ( $₫$ ) dorsal integument black, with evident metallic reflection. Body (Fig. 14) elongateoval $(\mathrm{LB}=2.35 \mathrm{~mm}$ ), moderately convex. Maximum pronotal width in middle: $0.68 \mathrm{~mm}$; maximum elytral width in middle: $1.03 \mathrm{~mm}$.

Head with impunctate vertex and frons, but with some coarse large punctures impressed near frontal grooves; surface slightly wrinkled. Frontal tubercles finely delimited, elongate, slightly oblique, with smooth surface; frontal grooves finely impressed, distally divergent from ocular margin; frontal carina both moderately widened and raised; labrum sub-trapezoidal, blackened; palpi strongly darkened; eyes sub-elliptical, normal size; antennae with reddish segments 1-3 and darkened segments 4-11; length of each segment of right antenna proportional to numerical sequence $24: 15: 14: 17: 20: 17$ : $20: 18: 20: 20: 26(1=0.032 \mathrm{~mm})$.

Pronotum slightly transverse $(\mathrm{LP}=0.50 ; \mathrm{WP} / \mathrm{LP}=$ $1.35)$ laterally slightly rounded, basally slightly narrower; lateral and basal margin finely bordered; punctation uniformly distributed with moderately but densely impressed small punctures on a reticulate surface. Scutellum wide, semicircular, with finely wrinkled surface.

Elytra elongate $(\mathrm{LE}=1.40 \mathrm{~mm} ; \mathrm{LE} / \mathrm{LP}=2.80)$, laterally rounded, apically obliquely sub-truncate; pygidium partially uncovered; punctation densely and uniformly distributed, but larger than pronotal punctures, impressed on a very smooth surface; humeral calli absent; subapterous wings.

Legs with yellowish tibiae and tarsi; third and fifth tarsomeres slightly darkened; proximal ventral edge of anterior and middle femora very weakly darkened; hind femora darkened; distal three quarters of hind tibiae dentate on external edge; apical spur of hind tibiae long about $3 / 5$ of tibial apex width, reddish; first anterior and middle tarsomeres weakly dilated. 
Ventral part entirely black; last sternite without dimples or special preapical impressions.

Median lobe of aedeagus (Fig. 23) moderately elongate $(\mathrm{LAED}=0.88 \mathrm{~mm}$; LE/LAED $=1.60)$, slender; in ventral view medially weakly narrower; apical part lanceolate, with an apically sub-rounded small median tooth; ventral sulcus elongate, apical third wide and then gradually narrows, basally not interrupted; dorsal sulcus V-shaped, apical third clearly visible; dorsal ligula very narrow; in lateral view median lobe slightly curved over almost its entire length with distinctly sinuous apical part.

Variation. ô $(\mathrm{n}=10$; mean and standard deviation $)$ : $\mathrm{LE}=1.42 \pm 0.18 \mathrm{~mm} ; \mathrm{WE}=1.03 \pm 0.10 \mathrm{~mm} ; \mathrm{LP}=0.53$ $\pm 0.05 \mathrm{~mm} ; \mathrm{WP}=0.70 \pm 0.07 \mathrm{~mm} ; \mathrm{LAN}=1.69 \pm 0.19$ $\mathrm{mm} ; \mathrm{LAED}=0.90 \pm 0.06 \mathrm{~mm} ; \mathrm{LB}=2.30 \pm 0.27 \mathrm{~mm}$; $\mathrm{LE} / \mathrm{LP}=2.70 \pm 0.16 ; \mathrm{WE} / \mathrm{WP}=1.48 \pm 0.05 ; \mathrm{WP} / \mathrm{LP}=$ $1.32 \pm 0.03 ; \mathrm{LE} / \mathrm{LAED}=1.57 \pm 0.12 ; \mathrm{LAN} /(\mathrm{LE}+\mathrm{LP})=$ $0.87 \pm 0.06$. ㅇ $(\mathrm{n}=10$; mean and standard deviation $)$ : $\mathrm{LE}$ $=1.66 \pm 0.17 \mathrm{~mm} ; \mathrm{WE}=1.24 \pm 0.08 \mathrm{~mm} ; \mathrm{LP}=0.56 \pm$ $0.05 \mathrm{~mm} ; \mathrm{WP}=0.78 \pm 0.07 \mathrm{~mm} ; \mathrm{LAN}=1.70 \pm 0.17 \mathrm{~mm}$; $\mathrm{LSP}=0.33 \pm 0.02 \mathrm{~mm} ; \mathrm{LB}=2.70 \pm 0.29 \mathrm{~mm} ; \mathrm{LE} / \mathrm{LP}=$ $3.00 \pm 0.09 ; \mathrm{WE} / \mathrm{WP}=1.60 \pm 0.08 ; \mathrm{WP} / \mathrm{LP}=1.41 \pm$ $0.06 ; \mathrm{LE} / \mathrm{LSP}=5.12 \pm 0.48 ; \mathrm{LAN} /(\mathrm{LE}+\mathrm{LP})=0.77 \pm$ 0.03 .

This species is variable in some chromatic characters: distal tarsomeres from pale to clearly darkened; anterior and middle femora from pale to partially, clearly darkened; antennae, especially first and distal antennomeres, from almost entirely pale to strongly darkened. Median lobe of aedeagus more or less elongate with ventral sulcus variable in width; in lateral view, median lobe more or less strongly arcuate. Females generally larger than males, with shorter antennae and first anterior and middle tarsomeres not enlarged. Spermatheca (Figs 33a-c) with sub-reniform, sometimes sub-cylindrical, basal part; distal part clearly developed, distinct from basal part; duct moderately elongate, sub-basally inserted, with one complete or incomplete coil.

Etymology. The name of this new species refers to the geographic region where it lives and means "from southern Africa".

Distribution. Western mountain areas of the Western Cape Province (Republic of South Africa) (Fig. 58). SouthernWestern Afrotropical chorotype (cf. Biondi \& D'Alessandro, 2006).

Host plants. Lobostemon cf. dorotheae, L. fruticosus, L. cf. trichotomus and Echium plantagineum (Boraginaceae).

\section{Longitarsus transvaalensis Biondi}

Longitarsus transvaalensis Biondi, 1999: 526.

Material examined. Republic of South Africa: Mpumalanga, Nelshoogte Pass, 1300-1600 m, 2549'S, 3048'E, 14.xii.1995 (forest edge), M. Biondi leg. 10, (holotype) (BAQ); ditto, 10 , 2 (paratypes) (BAQ).

Distribution. Mpumalanga Province (Republic of South Africa) (Fig. 56). Southern-Eastern Afrotropical chorotype (cf. Biondi \& D'Alessandro, 2006).

Host plants. This species was collected at forest edge on leaves of an unidentified plant belonging to the Borage family.

\section{KEY TO SPECIES}

This key identifies the 15 known Longitarsus species attributed to the capensis-group. However, as reported in Biondi (1999), it is not entirely useful for females since they lack reliable diagnostic characters within this species-group: in some species only the spermatheca shows some distinguishing characters, while the tignum, vaginal palpi, and $7^{\text {th }}$ tergite have no diagnostic value.

1 Hind femora yellowish. Antennae comparatively short [ 0 : $\mathrm{LAN} /(\mathrm{LE}+\mathrm{LP})<1.05 ;$ ㅇ LAN $/(\mathrm{LE}+\mathrm{LP})<0.96] \ldots . .2$

- Hind femora reddish, brownish or at least partially darkened; if hind femora yellowish then antennae clearly long $[$ đ̋: $\mathrm{LAN} /(\mathrm{LE}+\mathrm{LP})=1.05 ;$ ㅇ $\mathrm{LAN} /(\mathrm{LE}+\mathrm{LP})=0.96]$.

2 Third front tarsomere slightly wider than second (Fig. 55). Last abdominal sternite in male with a narrow median preapical longitudinal incision. Median lobe of aedeagus (Fig. 19) in ventral view tapered from base to apex and apically acute, without any evident median small tooth; ventral sulcus sub-parallel, wider at base. Spermatheca (Fig. 44) with broad collum not clearly distinct from basal part; duct uncoiled but clearly sinuous distally. .

L. neseri Biondi (Fig. 11$)$ .......................

Third front tarsomere clearly wider than second (Fig. 54). Last sternite in male without preapical impressions. Median lobe of aedeagus (Fig. 24) in ventral view slightly narrower in middle and apically widely rounded, with an evident median small tooth; ventral sulcus clearly narrower at base. Spermatheca (Fig. 41) with thin collum clearly distinct from basal part; duct uncoiled, not sinuous ............... L. cedarbergensis Biondi (Fig. 3)

3 Antennae long $(\widehat{\delta}: \mathrm{LAN} /(\mathrm{LE}+\mathrm{LP})=1.03 ;$ ㅇ $\mathrm{LAN} /(\mathrm{LE}+$ $\mathrm{LP})=0.96$ ). Third tarsomere clearly enlarged, about twice as wide as the second . . . . . . . . . . . . . . . . . 4 Antennae short ( 0 : LAN/(LE + LP) $<1.03 ;$ ㅇ LAN/(LE + LP) $<0.96)$. Third tarsomere generally narrow . . . . . . 5

4 Front and middle femora entirely pale; hind femora generally very weakly obscured. Elytra apically obliquely subtruncate (Fig. 52). Median lobe of aedeagus (Fig. 27) in ventral view sub-parallel, very slightly narrower in middle and apically sub-triangular; ventral sulcus wide, basally interrupted. Spermatheca (Fig. 38) generally with elongate basal part; duct uncoiled, sinuous proximally . ............ . . . . . . . . . . . . . . . . . L. malherbei sp.n. (Fig. 9) - Front and middle femora basally strongly darkened; hind femora clearly darkened. Elytra apically independently rounded (Fig. 53). Median lobe of aedeagus (Fig. 18) in ventral view clearly narrower in middle, apically sublanceolate; ventral sulcus less wide, basally not interrupted. Spermatheca (Figs 40a-b) generally with thicker basal part; duct uncoiled, sometimes sinuous proximally. ........... L. transvaalensis Biondi (Fig. 15)

5 Legs entirely pale but distal tarsi and hind femora brown. First three or four antennomeres entirely pale. Anterior angles of pronotum generally evident and spiky (Fig. 46). Inter-antennal space wide with frontal carina apically widely rounded (Fig. 46). Median lobe of aedegaus (Fig. 30) thickset, in ventral view apically widely and almost regularly rounded with a very weakly prominent small median tooth; dorsal ligula comparatively wide. Spermatheca (Figs $45 \mathrm{a}-\mathrm{b}$ ) with reniform basal part and well developed apical part; duct uncoiled, undulate or with a narrow coil . . . . . . . .................... L. grobbelaariae sp.n. (Fig. 5) 
- Legs differently coloured, darker and/or with not brown tarsi. First antennomeres generally darker. Anterior angles of pronotum generally scarcely evident and flattened. Interantennal space generally narrower or with frontal carina more flattened. Median lobe of aedeagus differently shaped, more elongate (Figs 16-17, 20-23, 25-26, 28-29) . . . . . 6

6 Pronotum less strongly transverse (generally WP/LP < 1.32), laterally very slightly rounded (Fig. 48). Elytral punctation less densely and deeply impressed (Fig. 48). Legs reddish, with slightly darkened hind femora. Antennae shorter $[0$ : $\mathrm{LAN} /(\mathrm{LE}+\mathrm{LP})=0.75 ;$ \& $\mathrm{LAN} /(\mathrm{LE}+\mathrm{LP})=0.72]$. Median lobe of aedeagus (Fig. 29) in ventral view weakly narrower in middle; apical part lanceolate, with a little median rounded tooth; ventral sulcus elongate, clearly narrower in middle; basally not interrupted. Spermatheca (Fig. $31 \mathrm{a}-\mathrm{c})$ bigger $(\mathrm{LSP}=0.39 \mathrm{~mm})$ with elongate and widely arcuate duct, uncoiled but sometimes sinuous distally . .... . . ................... . afromeridionalis sp.n. (Fig. 1)
Pronotum more strongly transverse, laterally clearly rounded (generally WP/LP $=1.32$ ). Elytral punctation more densely and deeply impressed. Legs differently coloured. Antennae generally longer [ $[\hat{0}: \mathrm{LAN} /(\mathrm{LE}+\mathrm{LP})>0.75$; 우 $\mathrm{LAN} /(\mathrm{LE}+\mathrm{LP})>0.72]$. Spermatheca smaller $(\mathrm{LSP}<0.39$

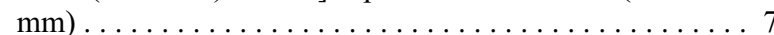

7 Pronotal punctation very densely and deeply impressed (Fig. 49). Front and middle femora at least partially darkened. First front and middle tarsomeres in male very clearly enlarged, sub-cylindrical, as long as second and third together and as wide as distal part of tibia (Fig. 47). Median lobe of aedeagus (Fig. 20) with apex broadly rounded, with an evident median apically rounded tooth; ventral sulcus wider in apical third then sub-parallel, basally not interrupted. Spermathecal duct (Fig. 39) always with a narrow coil. . . . . . . . . . . . L. melanicus Biondi (Fig. 10)

- Pronotal punctation generally sparser and/or less deeply impressed. Front and middle femora generally not darkened (sometimes obscured in L. sudafricanus sp.n.). First front and middle tarsomeres in male not or weakly enlarged and shorter than second and third together. .......... 8

8 Last sternite in male with a median longitudinal preapical incision. Median lobe of aedeagus (Fig. 22) with basally interrupted ventral sulcus; ventral sulcus very wide in apical third, convergent in middle part, narrow and sub-parallel basally; in lateral view, apical fifth of median lobe of aedeagus clearly and abruptly thinner than basal $4 / 5 \mathrm{~s}$. Spermathecal duct (Fig. 32) uncoiled, widely arcuate, with very short proximal part. . . . . . . . . L. rouxi sp.n. (Fig. 13)

- Last sternite in male without longitudinal incisions. Median lobe of aedeagus differently shaped. Spermathecal duct with long proximal part .................... 9

9 Last sternite in male with a wide and shallow oval median preapical dimple. Median lobe of aedeagus (Fig. 28) with basally interrupted ventral sulcus, medially with evident lateral sclerotized membranes. Spermathecal duct (Fig. 37) laterally inserted on basal part, uncoiled, widely arcuate ..... .

L. lugubris Biondi (Fig. 8)

- Last sternite in male without dimples or other special preapical impressions. Median lobe of aedeagus with differently shaped ventral sulcus. Spermathecal duct basally or sub-basally inserted on basal part . . . . . . . . . . . 10

10 Median lobe of aedeagus (Fig. 16) thickset, in ventral view sub-parallel; ventral sulcus short, only apical half visible, characterized by presence of a narrow, long and rather deep longitudinal groove. Spermatheca (Figs 34a-b) with generally short basal part; collum and apical part rather slender and clearly separate from basal part; duct generally uncoiled or with just a slight sign of a coil .. L. capensis Baly (Fig. 2) - Median lobe of aedeagus generally more slender, in ventral view more or less narrower in middle; ventral sulcus elongate, basally not interrupted, without evident longitudinal grooves. Spermatheca with more elongate basal part, and/or thick collum and apical part. . . . . . . . . . . . 11

11 Median lobe of aedeagus (Fig. 25) in ventral view apically broadly rounded with an evident median small tooth; basal half of ventral sulcus very narrow. Spermathecal duct (Fig. 42) thin, with a narrow coil .... L. luctuosus Biondi (Fig. 7) - Median lobe of aedeagus in ventral view apically more acutely rounded; median small tooth less evident; basal half of ventral sulcus less narrow. Spermathecal duct uncoiled or with a wider coil $\ldots \ldots \ldots \ldots \ldots \ldots \ldots \ldots \ldots \ldots$

12 First antenomere partially but distinctly darkened. Median lobe of aedeagus (Fig. 21) more slender, in ventral view apically not evenly rounded, with no evident median small tooth; in lateral view basal third weakly curved and then straight; ventral sulcus basally slightly narrower than apically. Spermathecal duct (Figs 35a-b) rather thin, distal tract sinuous or with a slight sign of a coil.

L. hexrivierbergensis sp.n. (Fig. 6)

- First antennomere not darkened. Median lobe of aedeagus in ventral view apically more evenly rounded, with an evident median small tooth; ventral sulcus basally clearly narrower than apically; in lateral view more strongly curved (Figs 17,

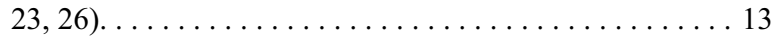

13 Median lobe of aedeagus (Fig. 17) shorter (LE/LAED = 1.71; LAED $=0.83 \mathrm{~mm}$ ), in lateral view with almost a straight apex. Spermathecal duct (Figs 36a-b) robust, uncoiled, narrowly arcuate ....... L. debiasei sp.n. (Fig. 4)

- Median lobe of aedeagus longer (LE/LAED = 1.68; generally LAED $>0.84 \mathrm{~mm}$ ), in lateral view with sinuous apex (Figs 23, 26). Spermathecal duct (Figs 33a-c, 43) at least sinuous or with a slight sign of a coil. ........... 14

14 Elytral punctation similar to that on pronotum (Fig. 50). Front and middle femora not darkened. Median lobe of aedeagus (Fig. 26) clearly slender, in lateral view apex abruptly bent in ventral direction. Spermathecal duct (Fig.

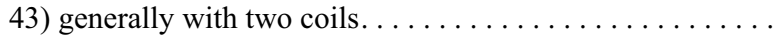
L. piketbergensis sp.n. (Fig. 12) Elytral punctation generally more deeply impressed than on pronotum (Fig. 51). Front and middle femora often partially darkened. Median lobe of aedeagus (Fig. 23) less slender, in lateral view apex more gradually bent in ventral direction. Spermathecal duct (Figs 33a-c) sinuous generally with one complete or incomplete coil. . L. sudafricanus sp.n. (Fig. 14)

\section{DISCUSSION}

Species of the capensis and anchusae groups are remarkably similar. In fact there is only one morphological trait, namely the degree of metathoracic wing reduction, that distinguishes the two groups; only subapterous species are known in the capensis species-group whereas brachypterous, sub-brachypterous or macropterous species can be found in the anchusae speciesgroup. The similarities, if shown to be synapomorphic by a future phylogenetic analysis, might suggest that the two groups comprise a monophyletic unit whose Mediterranean and South African subgroups became geographically separated relatively recently.

Alternative hypotheses could explain the separate distributions of Mediterranean and south African anchusae 
and capensis species-groups. As mentioned above, this particular type of distribution is widely documented for many plant and animal groups (cf. Axerold \& Raven, 1978; Balinsky, 1962; Coleman et al., 2003; Jürgens, 1997; La Greca, 1970, 1990). Excluding unlikely recent long-distance dispersal events, the most probable hypothesis to explain this type of geographical distribution is to accept there were ecological connections between the Mediterranean and South African areas in the past. Species in the capensis-group are significantly associated with "fynbos", a Mediterranean-type of vegetation that is widespread in the south-western part of South Africa and probably formed no earlier than the Late Miocene (cf. Axelrod \& Raven, 1978; Richardson et al., 2001). Based on this observation, the migratory flow of one or more Mediterranean capensis ancestors from the north towards South Africa may have occurred during the Quaternary period via "arid corridors" (sensu Balinski, 1962) which "appeared" in eastern Africa due to climatic changes that took place in the Northern Hemisphere during the glacial periods (cf. Jürgens, 1997).

In the Cape Province, the capensis species-group radiated into different species, often confined to rather limited areas (Figs 56-58). In the Mediterranean, during the Quaternary period, species diversification was very limited in the anchusae-group, as well as in other closely related species-groups (cf. Biondi, 1995). This occurred in many plant and animal groups in the Cape Province, which have a comparatively high biodiversities compared with ecologically similar areas. Different factors have been suggested to explain this phenomenon: (1) topographical complexity; (2) edaphic complexity; (3) pollinator specialization; (4) fire; and (5) short dispersal distance (Barraclough, 2006).

As mentioned above, species of the capensis-group are primarily associated with fynbos and plants of the genus Lobostemon (Boraginaceae). Lobostemon includes perennial shrubs with alternating leaves and flowers, mostly bell-shaped and usually pink or blue. This genus includes twenty-eight species endemic to South Africa and is largely confined to the winter rainfall area from Springbok to Mossel Bay and further eastward along the coast to near Grahamstown, where it rains throughout the year (Buys, 2006).

It is important to emphasize that 6 species of the 14 attributed to the capensis-group (Fig. 59) were collected from Echium plantagineum, an alien plant introduced into South Africa from Europe after 1825 and now present in Western and Eastern Cape and in the temperate mountain areas of Free State, Lesotho and Kwazulu-Natal, with some reports from southern Mpumalanga (cf. Retief \& Van Wyk, 1998). From a systematic point of view, the genus Echium is a sister-group of Lobostemon (Böhle et al., 1996), so the host-plant shift from Lobostemon to Echium observed in some species of the capensis-group could have easily occurred. However, in our opinion, this ecological event could dramatically alter the autecology and evolutionary mechanisms of the species involved. The presence of E. plantagineum in South Africa repre- sents, in fact, a new "shared" trophic resource for the sympatric species of the capensis-group and, simultaneously, an unexpected connection among allopatric species as consequence of the easy spread of this invasive plant. On the basis of other analogous adaptive processes observed in native species in response to the introduction of invasive plants (Mooney \& Cleland, 2001; Strauss et al., 2006), it is possible that unexpected evolutionary events could result in several different scenarios: (1) catalyze the local breakdown of reproductive isolation between native sympatric parent species; (2) bring into contact parent allopatric species and contribute to the reinforcement (or deletion) of reproductive isolating mechanisms; and (3) promote speciation by hybridization as an adaptive response of two or more native species to the new host-plant, as observed in species of the genus Rhagoletis Loew (Diptera Tephritidae) in testing invasive plants of Lonicera sp. (Caprifoliaceae) in North America (Schwarz et al., 2005, 2007).

ACKNOWLEDGEMENTS. We are grateful to the following colleagues who allowed us to study valuable material preserved in their respective institutions: S.L. Shute (BMNH), A. Vigna Taglianti (MCZR), E. Grobbelaar (SANC), R. Müller (TMSA) and $\mathrm{M}$. Uhlig and $\mathrm{H}$. Wendt (ZMHB). We would also like to offer special thanks to our friends, P.A. Audisio and A. De Biase (Italy, Rome), fellow travellers on many collecting trips in South Africa. This study was funded by grant PRIN 2004057217, "Zoogeography of Mediterranean-southern African disjunct distributions by a multi-method approach" from the Ministero dell'Università e della Ricerca. A professional scientific editor at Write Science Right was consulted in the preparation of this manuscript.

\section{REFERENCES}

Axelrod D.I. \& RAVEN P.H. 1978: Late Cretaceous and Tertiary vegetation history of Africa. In Werger M.J.A. \& Van Bruggen A.C. (eds): Biogeography and Ecology of Southern Africa. Junk Publishers, The Hague, pp. 77-130.

BALINSKY B.I. 1962: Patterns of animal distribution of the African continent. Ann. Cape Prov. Mus. 2: 299-310.

BALY J.S. 1877: Descriptions of new genera and uncharacterized species of Halticinae. Trans. Entomol. Soc. Lond. 10: 283-323.

BARRAClough T.G. 2006: What can phylogenetics tell us about speciation in the Cape flora? Divers. Distrib. 12: 21-26.

Berthold A.A. 1827: Latreille's natürliche Familien der Tierreichs, aus dem Französischen mit Anmerkungen und Zusätzen. Industrie Comptoirs, Weimar, $\mathrm{x}+604 \mathrm{pp}$.

Biondi M. 1995: The Longitarsus anchusae complex in Near Eastern and description of a new species (Coleoptera, Alticinae). Nouv. Rev. Entomol. (N.S.) 12: 259-271.

BIONDI M. 1996: Proposal for an ecological and zoogeographical categorization of the Mediterranean species of the flea beetle genus Longitarsus Berthold. In Jolivet P.H.A. \& Cox M.L. (eds): Chrysomelidae Biology. Vol. 3. General Studies. SPB Academic Publishing bv, Amsterdam, pp. 13-35.

BIONDI M. 1999: The black Longitarsus species associated with Boraginaceae in South Africa (Coleoptera, Chrysomelidae, Alticinae). In Cox M.L. (ed.): Advances in Chrysomelidae Biology 1. Backhuys Publishers, Leiden, pp. 515-531.

Biondi M. \& D'Alessandro P. 2006: Biogeographical analysis of the flea beetle genus Chaetocnema in the Afrotropical 
Region: distribution patterns and areas of endemism. J. Biogeogr. 33: 720-730.

Bryant G.E. 1941: New species of African Chrysomelidae (Col.). Proc. R. Entomol. Soc. Lond. 10: 209-214.

BuYs M.H. 2006: Lobostemon Lehm. At: http://www. plantzafrica.com/plantklm/lobostemon.htm.

Coleman M., Liston A., Kadereit J.W. \& Aвbott R.J. 2003: Repeat intercontinental dispersal and Pleistocene speciation in disjunct Mediterranean and desert Senecio (Asteraceae). Am. J. Bot. 90: 1446-1454.

Fernandez P. \& Hilker M. 2007: Host plant location by Chrysomelidae. Basic Appl. Ecol. 8: 97-116.

FURTH D.G. 1985: Some flea beetles and their foodplants from Kenya (Chrysomelidae: Alticinae). Coleopt. Bull. 39: 259-263.

Jolivet P.H.A. \& Hawkeswood T.J. 1995: Host-Plants of Chrysomelidae of the World. Backhuys, Leiden, $281 \mathrm{pp}$.

JÜRGENS N. 1997: Floristic biodiversity and history of African arid region. Biodiv. Conserv. 6: 495-514.

LA GrecA M. 1970: Considérations sur le peuplement animal terrestre de l'Afrique. C. R. Somm. Séan. Soc. Biogéogr. 409: $5-33$.

La GreCa M. 1990: Considerazioni sul popolamento animale dell'Africa Orientale. Biogeographia 14: 541-578.
Mooney H.A. \& Cleland E.E. 2001: The evolutionary impact of invasive species. Proc. Nat. Acad. Sci. 98: 5446-5451.

Retief E. \& VAN WYK A.E. 1998: The genus Echium (Boraginaceae) in southern Africa. Bothalia 28: 167-177.

Richardson J.E., Weitz F.M., FAy M.F., CRonk Q.C.B., Linder H.P., Reeves G. \& Chase M.W. 2001: Rapid and recent origin of species richness in the Cape Flora of South Africa. Nature 412: 181-183.

Schwarz D., Matta B.M., Shakir-Botteri N.L. \& McPheron B.A. 2005: Host shift to an invasive plant triggers rapid animal hybrid speciation. Nature 436: 546-549.

Schwarz D., Shoemaker K.D., Botteri N.L. \& McPheron B.A. 2007: A novel preference for an invasive plant as a mechanism for animal hybrid speciation. Evolution 61: 245-256.

Strauss S.Y., LaU J.A. \& Carroll S.P. 2006: Evolutionary responses of natives to introduced species: what do introductions tell us about natural communities? Ecol. Lett. 9: 357-374.

Verdú M., Pausas J.G., Segarra-Moragues J.G. \& Ojeda F. 2007: Burning phylogenies: fire, molecular evolutionary rates, and diversification. Evolution 61: 2195-2204.

WeISE J. 1895: Neue Chrysomeliden nebst synonymischen Bemerkungen. Dt. Entomol. Z. 2: 327-352.

Received February 1, 2008; revised and accepted May 6, 2008 